\title{
Rotational rest frequencies of the low lying vibrational states of $n$-propyl cyanide from extensive laboratory measurements up to $506 \mathrm{GHz}^{\star}$
}

\author{
Delong Liu ${ }^{1}$, Adam Walters ${ }^{1}$, Nadine Wehres $^{2}$, Frank Lewen ${ }^{2}$, Stephan Schlemmer ${ }^{2}$, and Holger S. P. Müller ${ }^{2}$ \\ 1 IRAP, Université de Toulouse, CNRS, CNES, UPS, 9 Av. Colonel Roche, BP 44346, 31028 Toulouse Cedex 4, France \\ e-mail: delong.liu@irap.omp.eu \\ 2 I. Physikalisches Institut, Universität zu Köln, Zülpicher Str. 77, 50937 Köln, Germany \\ e-mail: hspm@ph1.uni-koeln.de
}

Received 12 September 2018 / Accepted 9 December 2018

\begin{abstract}
Context. The spectra of four low-lying vibrational states of both anti and gauche conformers of normal-propyl cyanide were previously measured and analyzed in two spectral windows between 36 and $127 \mathrm{GHz}$. All states were then identified in a spectral line survey called Exploring Molecular Complexity with ALMA (EMoCA) toward Sagittarius B2(N) between 84.1 and $114.4 \mathrm{GHz}$ with the Atacama Large Millimeter/submillimeter Array (ALMA) in its Cycles 0 and 1.

Aims. We wanted to extend the measurements and analysis up to $506 \mathrm{GHz}$ to provide accurate predictions over a much wider range of frequencies, quantum numbers and energies.

Methods. We carried out measurements in two additional frequency windows up to $506 \mathrm{GHz}$.

Results. For the gauche conformer, a large number of both $a$ - and $b$-type transitions were identified. For the anti conformer, transitions were predominantly, but not exclusively, $a$-type. We hence improved molecular parameters for the ground states of both antiand gauche-n-propyl cyanide and for excited vibrational states of the gauche conformer $\left(v_{30}=1, v_{29}=1, v_{30}=2, v_{28}=1\right)$ and anti conformer $\left(v_{30}=1, v_{18}=1, v_{30}=2, v_{29}=1\right)$ with high order coupling parameters determined between $v_{18}=1$ and $v_{30}=2$. Parameters are published for the first time for $v_{18}=v_{30}=1$ of the anti conformer and for $v_{29}=v_{30}=1$ of the gauche conformer.

Conclusions. In total 15385 lines have been incorporated in the fits and should allow good predictions for unperturbed lines over the whole operating range of radio-telescopes. Evidence is found for vibrational coupling for some levels above $380 \mathrm{GHz}$. The coupling between $v_{18}=1$ and $v_{30}=2$ of the anti conformer has been well characterized. An additional list of 740 lines showing potential but as yet unidentified coupling has been provided for astrophysical identification.
\end{abstract}

Key words. molecular data - line: identification - astrochemistry - methods: laboratory: molecular - ISM: molecules submillimeter: ISM

\section{Introduction}

Molecules in vibrationally excited states are generally found in hot dense and possibly shocked regions of space and can be used as probes of the gas close to deeply embedded luminous infrared sources. For example, relatively simple molecules and linear carbon-chain molecules are found in the circumstellar envelopes of asymptotic giant branch stars. The following vibrationally excited molecules have been identified in IRC+10216, a mass-losing carbon star that is embedded in a thick dust envelope: HCN (Ziurys \& Turner 1986; Avery et al. 1994), $\mathrm{H}^{13} \mathrm{CN}$ (Groesbeck et al. 1994), SiS (Turner 1987a), CS (Turner 1987b), $\mathrm{C}_{4} \mathrm{H}$ (Guélin et al. 1987; Yamamoto et al. 1987). High lying vibrational states can be observed close to the photosphere, for example up to $10700 \mathrm{~K}$ for $\mathrm{HCN}$ (Cernicharo et al. 2011). A maser source of HCN originating in the doubly excited bending state was reported by Guilloteau et al. (1987) in CIT6 around $89 \mathrm{GHz}(J=1-0)$, and then IRC+10216 $(177 \mathrm{GHz}, J=2-1)$ by Lucas \& Cernicharo (1989). Schilke et al. (1999) reported a

* Line lists (Tables 10-25) are only available at the CDS via anonymous ftp to cdsarc.u-strasbg.fr (130.79.128.5) or via http: //cdsarc.u-strasbg.fr/viz-bin/qcat?J/A+A/622/A82 maser line originating in the quadruply excited bending state $(805 \mathrm{GHz}, J=9-8)$ in IRC+10216. A stronger line around $891 \mathrm{GHz}(J=10-9)$ in IRC+10216, CIT6 and Y CVn was later reported by Schilke \& Menten (2003). CIT6 and Y CVn are also mass-losing carbon stars. Simpler molecules may also be observed in protoplanetary nebula, for example $\mathrm{HCN}$ in CRL 618 (Thorwirth et al. 2003).

Vibrationally excited molecules including complex organic molecules, can also be found in star forming regions. The lowest energy vibrations are the easiest to detect and often include torsions. The first molecule detected in the ISM in a vibrationally excited state was cyanoacetylene (linear $\mathrm{HCCCN}$ ) in the Orion molecular cloud (Clark et al. 1976). HCN was detected in its bending vibration in Orion KL (Ziurys \& Turner 1986). Vibrationally excited ammonia $\left(\mathrm{NH}_{3}\right)$ was also detected toward Orion KL (Mauersberger et al. 1988; Schilke et al. 1992). Torsionally excited $\mathrm{O}$-bearing organic molecules identified include methanol $\left(\mathrm{CH}_{3} \mathrm{OH}\right)$ in Orion A (Lovas et al. 1982; Hollis et al. 1983), acetone $\left[\left(\mathrm{CH}_{3}\right)_{2} \mathrm{CO}\right]$ in Orion $\mathrm{KL}$ (Friedel et al. 2005), methyl formate $\left[\mathrm{HC}(\mathrm{O}) \mathrm{OCH}_{3}\right]$ in Orion $\mathrm{KL}$ (Kobayashi et al. 2007) and W51 e2 (Demyk et al. 2008). N-bearing organic molecules detected in a vibrationally excited state include formamide $\left(\mathrm{HCONH}_{2}\right)$ in Orion KL (Motiyenko et al. 2012), and 
alkyl cyanides that will be discussed later. The emission from complex organic molecules usually arises in compact regions, called hot cores, which are typically less than about $0.2 \mathrm{pc}$ in diameter (for example Mehringer et al. 2004). Hence the development of interferometers and in particular ALMA is creating a need for the spectra of the vibrational states (and isotopologues) of these molecules because of the increased sensitivity, and column density due to the decreased beam size.

The study of vibrationally excited states of known molecules in space has several astrophysical interests. Firstly, the frequencies of these lines need to be known so as to make a complete spectroscopic model of an object, identify all lines due to known molecules and hence be able to focus on remaining lines as candidates for new unidentified species (see for example, Mehringer et al. 2004; Daly et al. 2013). Secondly, work on the vibrational states can be used to take the latter into account in the partition function and hence to better estimate the column density of the molecule observed (for example Müller et al. 2016). Thirdly, lines of molecules in vibrationally excited states can be used to focus on hotter, or shocked regions of an object such as hot cores in star-forming regions and to model the physical and chemical properties of these regions (for example Goldsmith et al. 1983; Ziurys \& Turner 1986; Mehringer et al. 2004). Applications include determining vibrational temperatures to check whether they are in equilibrium (for example Motiyenko et al. 2012) exploring infrared pumping (for example Schilke \& Menten 2003; Belloche et al. 2013) and determining the temperature of dust in the cores (Schilke et al. 1992).

Methyl cyanide, the simplest alkyl cyanide, is among the molecules detected early by radio astronomy (Solomon et al. 1971) and an unknown line at $92.3527 \mathrm{GHz}$ observed in Orion and toward the Sagittarius B2 molecular cloud complex (denoted Sgr B2) was suggested to be due to this molecule in its lowest $v_{8}=1$ vibrational state as early as 1976 (Clark et al. 1976). Then Goldsmith et al. (1983), using the Five College Radio Astronomical Observatory, modeled 12 components of the $J=6-5$ transition around $111 \mathrm{GHz}$ to confirm the detection of vibrationally excited $\mathrm{CH}_{3} \mathrm{CN}$ in its lowest $v_{8}=1$ state, a degenerative bending mode at around $525 \mathrm{~K}$ equivalent energy, toward the central region of Orion. Using early science verification data from the Atacama Large Millimeter/submillimeter Array (ALMA), the higher state of $v_{8}=2$ at around $1030 \mathrm{~K}$ was also detected in the hot core of Orion KL (Fortman et al. 2012). Belloche et al. (2013) published a complete IRAM $30 \mathrm{~m}$ line survey of Sgr B2(N) and (M), through which, they detected both $v_{8}=1$ and 2 states of methyl cyanide in the two sources; as well as that, of a higher state $v_{4}=1$ at around $1320 \mathrm{~K}$ and for the first time transitions of $v_{8}=1{ }^{13} \mathrm{C}$ substituted methyl cyanide in $\mathrm{Sgr}$ $\mathrm{B} 2(\mathrm{~N})$.

The first publication of an observation of vibrationally excited ethyl cyanide was by Gibb et al. (2000). Transitions of its two lowest-lying states $\left(v_{13}=1\right.$ in-plane bending mode and $v_{21}=1$ methyl torsional mode both around $300 \mathrm{~K}$ ) were observed toward the organic-rich hot core G327.3-0.6 using the Swedish-ESO Submillimetre Telescope. A paper devoted to the detection of vibrationally excited ethyl cyanide in the Sgr B2 Large Molecule Heimat source (Sgr B2(N-LMH)) was published by Mehringer et al. (2004). By using the Caltech Submillimeter Observatory, in the range $215-270 \mathrm{GHz}$, the Berkeley-IllinoisMaryland Association Array and the Caltech Millimeter Array in the $107-114 \mathrm{GHz}$ range, the authors detected several lines from these two vibrationally excited states that lie about $302 \mathrm{~K}$ above the ground state. Higher excited states of ethyl cyanide, $v_{20}=1$ at around $530 \mathrm{~K}$ and $v_{12}=1, \mathrm{CCC}$ bending state at around
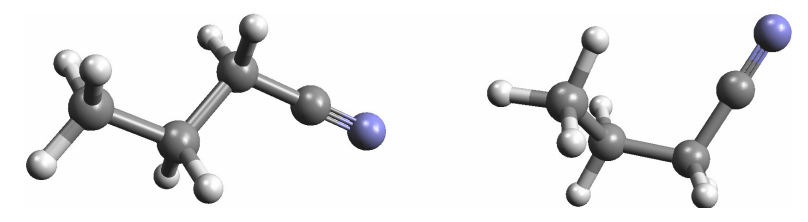

Fig. 1. Schematic depiction of the anti (left) and the gauche (right) conformers of $n$-PrCN. The $\mathrm{C}$ and $\mathrm{N}$ atoms are represented by gray and violet "spheres" respectively, and the $\mathrm{H}$ atoms by small, light gray ones.

$760 \mathrm{~K}$, were first detected toward three hot cores of Orion KL by Daly et al. (2013), along with the two other lower states. Ethyl cyanide in all these four states was also detected in Sgr B2(N) and its lowest states $\left(v_{13}=1\right.$ and $\left.v_{21}=1\right)$ in Sgr B2(M) by Belloche et al. (2013).

There are two isomers of propyl cyanide (also known as cyanopropane or butyronitrile), the straight-chain normal-, $n$-propyl cyanide (here $n$-PrCN for short), and the branched $i$ iso-, $i$-propyl cyanide (here $i$-PrCN for short). Early laboratory work on the ground state of $i$-PrCN (Herberich 1967; Durig \& Li 1974) was extended to frequencies needed for radioastronomy by Müller et al. (2011). This isomer became the first molecule found in the interstellar medium with a branched carbon backbone (Belloche et al. 2014). Recently, very comprehensive work on the laboratory measurements and analysis of vibrationally excited states of $i$-PrCN was reported (Kolesniková et al. 2017). There are two conformers of $n$-propyl cyanide as schematically depicted in Fig. 1: anti- (here $a-n$-PrCN for short) with a planar heavy atom frame (i.e., a dihedral CCCC angle of $180^{\circ}$ ); and gauche- (here $g$ - $n$ - $\mathrm{PrCN}$ for short) in which the $\mathrm{CH}_{3}$ group, or equivalently the $\mathrm{CN}$ group, is rotated by $\sim 120^{\circ}$ to form a dihedral CCCC angle of about $\pm 60^{\circ}$.

Belloche et al. (2009) identified $a-n-\mathrm{PrCN}$ (in its ground vibrational state) for the first time toward Sgr B2(N) a site of high-mass star formation, in a line survey using the IRAM $30 \mathrm{~m}$ telescope. Lines of the gauche conformer could be included correctly in their model but were too blended for a conclusive identification. Transitions of $g-n$-PrCN were later detected unambiguously along with the detection of $i$-PrCN. These identifications were made in a spectral line survey called Exploring Molecular Complexity with ALMA (EMoCA; Belloche et al. 2016). This survey, between 84.1 and $114.4 \mathrm{GHz}$ was taken with ALMA toward Sgr B2(N). Müller et al. (2016), using the same survey, reported the identification of four vibrationally excited states for both anti- and gauche- $n$-PrCN following new spectroscopic work summarized in the last paragraph.

Laboratory measurements of $n$-PrCN in the vibrational ground states have been sufficient to detect this molecule in space for some time, however, it is only recently that predictions of some of the vibrational states have been good enough to envisage their detection. The ground state rotational constants of $n$-PrCN were first reported by Hirota (1962) following measurements up to $32 \mathrm{GHz}$. Demaison \& Dreizler (1982) and Vormann \& Dreizler (1988) used Fourier transform microwave spectroscopy to study the ${ }^{14} \mathrm{~N}$ quadrupole structure up to $26 \mathrm{GHz}$. The latter authors also studied the methyl internal rotation. Wlodarczak et al. (1988) extended the measurements up to $300 \mathrm{GHz}$ and measured the dipole moment components. The energy difference between the two conformers is small leading to some confusion as to that of lower energy. The previous authors determined from intensity measurements that the anti conformer is lower in energy than the gauche by $1.1 \pm 0.3 \mathrm{~kJ} \mathrm{~mol}^{-1}$. Durig et al. (2001) used infrared spectroscopy of $n$-PrCN dissolved in liquid Xenon to determine that the gauche conformer 
is lower than the anti by $0.48 \pm 0.04 \mathrm{~kJ} \mathrm{~mol}^{-1}$ (or $58 \pm 4 \mathrm{~K}$ ) and Müller et al. (2016) found this value to be fully consistent with their model of the ALMA spectra.

Hirota (1962) also determined the rotational constants of the three lowest fundamental vibrational states of the anti and gauche conformers. The information of these excited states for both conformers is summarized in Table 1 . Since no gas phase measurements are available the vibrational frequencies given are scaled $a b$ inito values from Durig et al. (2001). The nomenclature of the states differs from that in the aforementioned publication since although the gauche conformer has $C_{1}$ symmetry, with all vibrations belong to the symmetry class $a$; the anti conformer has $C_{S}$ symmetry with 18 fundamental vibrations belonging to the symmetry class $a^{\prime}$ and 12 to the symmetry class $a^{\prime \prime}$ (Crowder 1987). The equivalent energy for the $a$ - $n$-PrCN includes the energy of this conformer above that of the gauche. As can be seen from the equivalent temperatures in Table 1, these vibrational states are predicted to be substantially populated in hot core regions of star-formation where temperatures can rise to around $100-300 \mathrm{~K}$. For comparison the lowest vibrational states are around $525 \mathrm{~K}$ for methyl cyanide, $302 \mathrm{~K}$ for ethyl cyanide, as detailed above, and $266 \mathrm{~K}$ for $i$-PrCN. Recently, the laboratory spectroscopic study up to $127 \mathrm{GHz}$ of these vibrationally excited states (Müller et al. 2016) led to their detection in space as explained above. Following this identification we decided to carry out a more extensive analysis up to $506 \mathrm{GHz}$ of these vibrational states with the aim of providing reliable predictions over the whole operating band of ALMA. The present data should be useful to search for vibrationally excited states of $n$-PrCN in Orion KL where transitions of the ground vibrational states of both conformers were detected recently with ALMA (Pagani et al. 2017). During our work we also extended the spectral analysis of the ground state, and carried out new work on the combination states of $v_{18}=v_{30}=1$ for the anti conformer and $v_{29}=v_{30}=1$ for the gauche conformer. Transitions of these and other higher-lying vibrational states may be observable in the new EMoCA data obtained in ALMA Cycle 4.

\section{Laboratory spectroscopic details}

All measurements were carried out at Universität zu Köln. A schematic diagram of the experimental arrangement is shown in Fig. 2. The experimental arrangement for measurements between $36-70 \mathrm{GHz}$ and between $89.25-126.75 \mathrm{GHz}$ have been described previously (Müller et al. 2016). In the two new higher frequency ranges (171-251 and 310-506 GHz) we used a $5.1 \mathrm{~m}$ long double path (10.2 $\mathrm{m}$ total) absorption cell with inner diameter of $100 \mathrm{~mm}$ and equipped with Teflon windows. A commercial sample of $n$-PrCN was flowed slowly through the cell at pressures of around $1 \mathrm{~Pa}$. The cell was at room temperature but the inlet system was heated to about $50^{\circ} \mathrm{C}$ to achieve a stable pressure in the cell and prevent condensation blocking the needle valve used for flow adjustment. Measurements between $171-251 \mathrm{GHz}$ and between $310-506 \mathrm{GHz}$ used a Virginia Diodes WR9.0 THz starter kit with cascaded multipliers and respectively $3 \mathrm{~mW}$ and $0.18 \mathrm{~mW}$ middle range output power. Toward the edges the power is around a factor of 15 smaller. This chain was driven by a Rohde \& Schwarz SMF 100A synthesizer. From $171-251 \mathrm{GHz}$ eighteen times multiplication and $63 \mathrm{kHz}$ point spacing was used, from $310-506 \mathrm{GHz}$ thirty six times and $144 \mathrm{kHz}$. We carried out large spectral scans of around $6-7 \mathrm{GHz}$ taking typically several hours to acquire. Up and down scans were co-added. Schottky diode detectors were used to detect output power. We used frequency modulation (FM) throughout

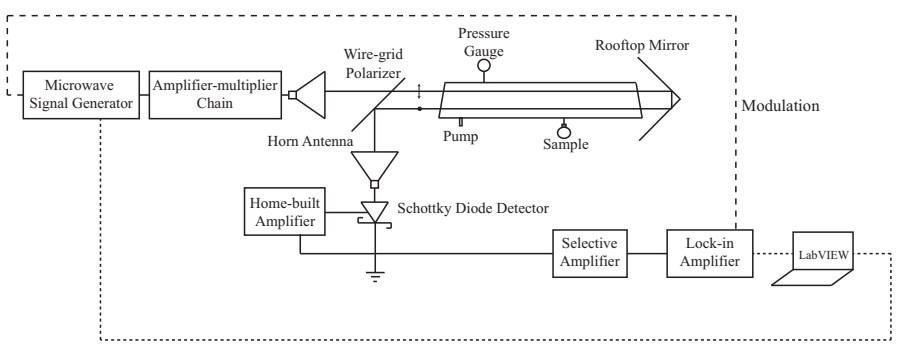

Fig. 2. Setup diagram for the molecular absorption spectra measurements. The arrow and dot between the wire-grid polarizer and tilted window express the polarizations of the incident and outgoing radiation.

with demodulation at $2 f$, which causes an isolated line to appear approximately as a second derivative of a Gaussian. Optimally the FM deviation was set to half the linewidth, hence typically $250 \mathrm{kHz}$ around $300 \mathrm{GHz}$. The sensitivity of the spectrometer systems varied with frequency showing both a diminution on the edges of the frequency bands and fluctuations throughout (source power, coupling, reflections, absorption by the optics) in spite of periodic optimization. Nevertheless, relative intensities could be used as guidance for assignments by comparing lines relatively close in frequency.

\section{Spectroscopic results and discussion}

\subsection{Ground vibrational state}

The frequency of previous fits of the ground states was limited to $300 \mathrm{GHz}$. Our latest fits of the vibrationally excited states were based on deviations from the ground-state parameters and were made up to $506 \mathrm{GHz}$. Hence we found it useful to also include new higher frequency measurements and make an up-dated fit of the ground state. The extension of the fit, and the increasing precision of predictions allowed us to also include 1002 additional transitions for the anti conformer and 1545 for the gauche in the range below $300 \mathrm{GHz}$, including $b$ type and hyperfine split transitions. The assigned uncertainties were $0.01-0.1 \mathrm{MHz}$, depending on the quality of lines. Usually uncertainties of $0.05-0.1 \mathrm{MHz}$ were assigned above $310 \mathrm{GHz}$ because of the very crowed spectrum which is caused for example by the increase of lines for a given $J$ and the increase in line width (to more than $1 \mathrm{MHz}$ ). The difference in the components of the dipole moment for the anti and gauche conformers has an implication on the type of transitions that can be measured and on the parameters that can be determined as will be elaborated below. We took the following dipole moment components for our predictions: for $a-n-\operatorname{PrCN}, \mu_{a}=4.0 \mathrm{D}$, $\mu_{b}=0.98 \mathrm{D}, \mu_{c}=0$ (by symmetry); for $g-n-\operatorname{PrCN}, \mu_{a}=3.27 \mathrm{D}$, $\mu_{b}=2.14 \mathrm{D}, \mu_{c}=0.45 \mathrm{D}$. Most of the values are those determined by Wlodarczak et al. (1988), however, $\mu_{a}$ for $a-n-\operatorname{PrCN}$, and $\mu_{c}$ for $g-n-\operatorname{PrCN}$ were taken from quantum-chemical calculations made by H.S.P Müller using the method described in Müller et al. (2011). The value of $\mu_{a}$ for $a-n-\operatorname{PrCN}$, some $10 \%$ larger than that quoted by Wlodarczak et al. (1988) is also consistent with astrophysical observations (Belloche et al. 2014).

In total we added 1992 new measured transitions (1284 lines because of transitions close or at the same frequency) for the anti conformer in the frequency bands of 36-70, 89-127, $171-251$ and $310-506 \mathrm{GHz}$ with $K_{a}$ up to 29 and $J$ up to 115 for the lower state (denoted $K_{a}^{\prime \prime}$ and $J^{\prime \prime}$ ). The fitted lines for the anti conformer are shown in Table 10 (available at the CDS, 
Table 1. Lowest fundamental vibrational states of $n$-PrCN.

\begin{tabular}{cccccc}
\hline \hline \multirow{2}{*}{$g / a$} & \multirow{2}{*}{ Description } & \multicolumn{2}{c}{$g$ - $n$-PrCN } & \multicolumn{2}{c}{$a-n$-PrCN } \\
\cline { 3 - 5 } & & Frequency $\left(\mathrm{cm}^{-1}\right)$ & Equivalent $T(\mathrm{~K})$ & Frequency $\left(\mathrm{cm}^{-1}\right)$ & Equivalent $T(\mathrm{~K})$ \\
\hline$v_{30 / 30}$ & Asymmetric torsion & 116 & 167 & 101 & 203 \\
$v_{29 / 18}$ & $\mathrm{C}-*$ C-C bend & 179 & 258 & 163 & 293 \\
$v_{28 / 29}$ & $\mathrm{CH}_{3}$ torsion & 262 & 377 & 241 & 405 \\
\hline
\end{tabular}

Notes. $g / a$ gives the nomenclature for the gauche/anti conformer respectively. The frequencies are ab initio values taken from Durig et al. (2001). For the anti confomer the equivalent temperature also includes the energy of this conformer ( $58 \mathrm{~K}$ equivalent) above that of the gauche. ${ }^{*} \mathrm{C}$ represents the $\mathrm{CH}_{2}$ group adjacent to the $\mathrm{C} \equiv \mathrm{N}$.

contains the following information: rotational transition represented by quantum numbers of upper and lower levels, assigned frequency, calculated frequency, residual (assigned-predicted), uncertainty assigned; and for non-resolved transitions: weighted average predicted frequency and difference from assigned frequency). In these newly assigned lines, there were 154 hyperfine split transitions and all of them could be fitted with the hyperfine structure parameters from Vormann \& Dreizler (1988). The hyperfine split lines were all below $100 \mathrm{GHz}$, because of the increasing overlap and broadening of lines at higher frequencies. Besides mainly $a$-type transitions, $20 b$-type lines were assigned and fitted. All transitions were $R$-branch because of their stronger intensities in $a-n-\operatorname{PrCN}$. The assigned $b$-type $R$-branch lines with $\left(\Delta J, \Delta K_{a}, \Delta K_{c}\right)=(1, \pm 1,1)$ were in the frequency below $251 \mathrm{GHz}$ and have a $K_{a}^{\prime \prime}$ up to 2 and $J^{\prime \prime}$ up to 55 . Using the larger set of fitted lines, we were able to refine the molecular parameters which are listed in Table 2 together with the parameters determined in Belloche et al. (2009). By comparison we can see that uncertainties on the parameters are smaller. However, the WRMS of our fits is somewhat higher as it is close to or slightly less than 1 , which indicates experimental uncertainties are comparable to residuals. We were also able to add some octic centrifugal distortion parameters $\left(L_{K K J}, L_{J J K}\right.$ and $\left.L_{J}\right)$. We constrained the value of $H_{K}$ to the same value as its estimation in Belloche et al. (2009) since it could not be correctly fit because of the persistent lack of $b$-type transitions with higher values of $K_{a}$.

We give some additional information to quantify the reliability of predictions. For $R$-branch $a$-type transitions the limit of good predictions is $J^{\prime \prime}=123$ at $K_{a}^{\prime \prime}=32$. This limit is specified for uncertainties on the predictions less than $0.1 \mathrm{MHz}$ and a similar criterion will be used for the following sections. For lower $K_{a}$ the limit in $J$ increases slowly and for higher $K_{a}$ it decreases rapidly as is the case for the examples that will be cited for the other conformer and other vibrational states.

For the gauche conformer, because of its more asymmetrical geometry and larger $b$-component of the dipole moment, more different types of transition (compared to the anti) were intense enough to be identified and assigned. Totally 3290 more transitions (1861 new lines) were fitted and shown in Table 11 (available at the CDS); they are $1318 R$-branch $a$-type transitions, $143 Q$-branch $a$-type transitions, $1255 R$-branch $b$-type transitions, $448 Q$-branch $b$-type transitions and $126 P$-branch $b$-type transitions. All of the newly assigned $Q$ - and $P$ - branch lines are below $320 \mathrm{GHz} .442$ hyperfine split transitions were included and fitted with parameters from Vormann \& Dreizler (1988). Both $a-n$-PrCN and $g$ - $n$-PrCN showed prolate pairing. Again due to the more asymmetrical geometry of $g-n$-PrCN only $K_{a}^{\prime \prime}=0$ and 1 lines could be found oblate paired for the anti conformer whereas for the gauche conformer oblate pairing could be observed up to between $K_{a}^{\prime \prime}=7$ and 8 . The newly fitted lines of $g-n$-PrCN also allowed us to refine the parameter list as shown in Table 2, with all parameters showing uncertainties at least ten times lower than those quoted in Belloche et al. (2009). Additionally, higher order centrifugal distortion parameters: $L_{J J K}, L_{J}$, $l_{1}$ and $P_{K K K J}$ could be determined. As regards good predictions (see the anti conformer) the limit of $R$-branch $a$-type transitions is for example $J^{\prime \prime}=99$ at $K_{a}^{\prime \prime}=50$. Considering oblate pairing at high frequencies, transitions with $\left(\Delta J, \Delta K_{a}, \Delta K_{c}\right)=(1,1,1)$ were used to make confident predictions. Finally, all $b$-type transitions at frequencies below $950 \mathrm{GHz}$ were well predicted with $K_{a}^{\prime \prime}$ up to 34 .

\section{2. $v_{30}=1$ and 2 of $g-n-\operatorname{PrCN}$}

$v_{30}=1$ is the lowest-lying vibrationally excited state of $g-n$-PrCN which corresponds to a $\mathrm{C}_{2} \mathrm{H}_{5}$ torsion around the central C atom (Crowder 1987; Müller et al. 2016). In this work, measurements at $171-251$ and $310-506 \mathrm{GHz}$ were added to refine the parameters; the frequency of transitions was limited to $127 \mathrm{GHz}$ in Müller et al. (2016). We assigned new lines in the sequence of increasing $J$ for each $K_{a}$ first in the lower frequency range and then for the higher one, so as to carefully check the coherence of the evolution of molecular parameters. When new lines could not be fit within experimental uncertainties, and especially when trends were observed, new additional parameters were tried sequentially. The final derived parameters with their uncertainties are listed in Table 3 and those determined in Müller et al. (2016) are listed simultaneously as comparison. Lines of $v_{30}=1$ and $v_{30}=2$ were fit together. We followed the method for coding the parameters of vibrationally excited states used in previous papers, that is $\Delta X$ represents $X_{v=1}-X_{v=0}$, and $\Delta \Delta X=X_{v=2}-X_{v=0}-2 \Delta X$. Using this method, we took advantage of the relation between vibrational states to reduce the number of parameters needed to be determined. Furthermore, $\Delta X$ should be significantly smaller than $X_{v=0}$, and $\Delta \Delta X$ significantly smaller than $\Delta X$, giving a useful check on parameters with abnormal values. The decrease in intensities of lines of higher excited states made their fitting more difficult, especially at high frequency. Finally we could fit 2632 more transitions (1524 new lines) for $v_{30}=1$. All of the newly fitted 1457 $a$-type transitions were $R$-branch with $\Delta K_{a}=0$; we tried to find $Q$-branch $a$-type lines but they were much weaker (for example for two lines compared around $178.9 \mathrm{GHz}$ the $Q$-branch $a$-type transition was about 50 times weaker than a nearby $R$-branch $a$-type transition). The highest $J^{\prime \prime}$ of $a$-type lines we could fit in our available frequency was around 90 near $500 \mathrm{GHz}$, with somewhat higher values for low $K_{a}$ and somewhat lower values for high $K_{a}$. We fitted $a$-type transitions with $K_{a}^{\prime \prime}$ up to 20 over all ranges, and an additional 32 transitions with $K_{a}^{\prime \prime}$ between 21 
D. Liu et al.: Frequencies of the low lying vibrational states of $n$-propyl cyanide up to $506 \mathrm{GHz}$

Table 2. Molecular parameters for the ground vibrational states of $n$-PrCN obtained from our latest fit using Watson's $S$ reduction compared to the fit of Belloche et al. (2009).

\begin{tabular}{|c|c|c|c|c|}
\hline \multirow[t]{2}{*}{ Parameters(MHz) } & \multicolumn{2}{|c|}{$g-n-\operatorname{PrCN}$} & \multicolumn{2}{|c|}{$a-n-\operatorname{PrCN}$} \\
\hline & (Belloche et al. 2009) & This work & (Belloche et al. 2009) & This work \\
\hline$A$ & $10060.41649(108)$ & $10060.41587(11)$ & 23668.31931(143) & $23668.31883(44)$ \\
\hline$B$ & $3267.662408(301)$ & $3267.662256(28)$ & $2268.146892(147)$ & $2268.146732(27)$ \\
\hline$C$ & $2705.459572(290)$ & $2705.459402(24)$ & $2152.963946(168)$ & $2152.963902(27)$ \\
\hline$D_{K} \times 10^{3}$ & $60.235(6)$ & $60.2384(5)$ & $240.653(29)$ & $240.571(14)$ \\
\hline$D_{J K} \times 10^{3}$ & $-18.26470(117)$ & $-18.26546(9)$ & $-10.82631(92)$ & $-10.82269(21)$ \\
\hline$D_{J} \times 10^{6}$ & $3195.064(207)$ & $3194.979(15)$ & $398.674(69)$ & $398.722(8)$ \\
\hline$d_{1} \times 10^{6}$ & $-1037.470(55)$ & $-1037.503(5)$ & $-46.637(42)$ & $-46.5923(23)$ \\
\hline$d_{2} \times 10^{6}$ & $-77.1864(183)$ & $-77.1647(16)$ & $-0.5901(59)$ & $-0.5889(17)$ \\
\hline$H_{K} \times 10^{6}$ & $1.806(18)$ & $1.8411(9)$ & $2.5^{a}$ & $2.5^{a}$ \\
\hline$H_{K J} \times 10^{9}$ & $-517.3(35)$ & $-518.81(18)$ & $372.4(24)$ & $422.1(8)$ \\
\hline$H_{J K} \times 10^{9}$ & $9.92(68)$ & $9.804(27)$ & $-20.67(20)$ & $-21.600(25)$ \\
\hline$H_{J} \times 10^{9}$ & $4.486(56)$ & $4.4445(33)$ & $0.353(11)$ & $0.3743(9)$ \\
\hline$h_{1} \times 10^{9}$ & $2.505(29)$ & $2.5205(16)$ & $0.117(14)$ & $0.09830(15)$ \\
\hline$h_{2} \times 10^{12}$ & $524.6(135)$ & $518.38(26)$ & & $1.83(12)$ \\
\hline$h_{3} \times 10^{12}$ & $111.3(31)$ & $109.02(10)$ & & \\
\hline$L_{K K J} \times 10^{12}$ & $30.6(31)$ & $32.47(20)$ & & $-72.7(8)$ \\
\hline$L_{J K} \times 10^{12}$ & $-4.11(78)$ & $-4.209(18)$ & & \\
\hline$L_{J J K} \times 10^{15}$ & & $118.40(308)$ & & $35.55(131)$ \\
\hline$L_{J} \times 10^{15}$ & & $-2.273(246)$ & & $-0.513(34)$ \\
\hline$l_{1} \times 10^{15}$ & & $-2.013(134)$ & & \\
\hline$P_{K K K J} \times 10^{15}$ & & $-0.807(60)$ & & \\
\hline$\chi_{a a}$ & & $-1.683^{b}$ & & $-3.440^{b}$ \\
\hline$\chi_{b b}$ & & $-0.252^{b}$ & & $1.385^{b}$ \\
\hline$\chi_{c c}$ & & $1.935^{b}$ & & $2.055^{b}$ \\
\hline WRMS & 0.66 & 0.98 & 0.75 & 0.97 \\
\hline Highest frequency & 282073 & 499969 & 283196 & 499936 \\
\hline $\operatorname{Max} J^{\prime \prime}$ & 47 & 91 & 63 & 115 \\
\hline $\operatorname{Max} K_{a}^{\prime \prime}$ & 34 & 45 & 23 & 29 \\
\hline Transitions & 215 & 3505 & 207 & 2199 \\
\hline
\end{tabular}

Notes. The fits of Belloche et al. (2009) are based on work from Demaison \& Dreizler (1982), Wlodarczak et al. (1988), Vormann \& Dreizler (1988). WRMS is short for weighted root mean square used to evaluate the final fit. WRMS $=\sqrt{\frac{\sum_{i}\left(\frac{\delta(i)}{\operatorname{err}(i)}\right)^{2}}{N}}$, where $\delta(i)$ is the residual of each transition assigned; $\operatorname{err}(i)$ is the uncertainty evaluated; $N$ is the number of transitions. Numbers in parantheses represent the uncertainty compared to the last quoted digit. ${ }^{(a)}$ The value of $H_{K}$ was estimated by Belloche et al. (2009). ${ }^{(b)}$ The new added hyperfine split lines can all be fit correctly with the hyperfine structure parameters from Vormann \& Dreizler (1988), so they were fixed in this work.

and 25 between $171-251 \mathrm{GHz}$. Like in the ground state, $a$-type high $J$ low $K_{a}$ transitions were usually oblate paired with $b$-type transitions in the same branch. Totally $1175 b$-type transitions in both $R$ - and $Q$-branches were added to the fit. Most $b$-type transitions assigned were $R$-branch and more occurred in the high frequency range as the energy between neighboring $K_{a}$ levels gets larger quickly with increasing $K_{a}$. Among the newly fitted $R$-branch $b$-type transitions about half had $\left(\Delta J, \Delta K_{a}, \Delta K_{c}\right)=$ $(1,1,1)$ with $K_{a}^{\prime \prime}$ covering 0 to 19 , while the transitions with $(1,-1,1)$ all had $K_{a}^{\prime \prime}$ up to 12 and the transitions with $(1,1,-1)$ had $K_{a}^{\prime \prime}$ not less than 6. Among the $Q$-branch, previously fitted transitions have $K_{a}^{\prime \prime}$ less than 12 between $37.30-125.54 \mathrm{GHz}$, and 204 newly fitted transitions were all $b$-type with $K_{a}^{\prime \prime} \leq 12$ between 171-251 GHz. The refined parameters enable good predictions (see Sect. 3.1) of $R$-branch $a$-type transitions up to $J^{\prime \prime}=91$ at $K_{a}^{\prime \prime}=30$, and $b$-type transitions with $\left(\Delta J, \Delta K_{a}, \Delta K_{c}\right)=(1,1,1)$ up to $J^{\prime \prime}=95$ at $K_{a}^{\prime \prime}=20$.

Fitted transitions of $v_{30}=2$ above $171 \mathrm{GHz}$ were similar in type to those of $v_{30}=1: 1045 R$-branch $a$-type transitions,
$778 R$-branch $b$-type transitions, and $214 Q$-branch $b$-type transitions; with no new hyperfine split transitions. Table 12 (available at the CDS) gives all lines of $v_{30}=1$ and $v_{30}=2$ used in the final fit. We found it was difficult to fit $a$-type transitions with $J^{\prime \prime}$ larger than 89 for $K_{a}^{\prime \prime}=0$ and 1 , as all these transitions showed residuals (assigned-predicted) with a trend of increasing negative values. New parameters could not be determined to remove this trend. Therefore we put all these identified transitions in a separate list (Table 13, at the CDS). As a precaution, all same-type transitions with $J^{\prime \prime}$ larger than 89 for higher $K_{a}^{\prime \prime}$ were put into this supplementary list even if the lines appeared to fit correctly. An obvious trend of residuals also took place with $J^{\prime \prime}$ larger than 59 for $K_{a}^{\prime \prime}=20$. The limit of safe $a$-type lines climbs to $K_{a}^{\prime \prime}=22$ with $J^{\prime \prime}$ up to 58 . $R$-branch $b$-type transitions in our newly assigned frequency ranges could be classified in 3 groups as was the case for $v_{30}=1$ : transitions with $\left(\Delta J, \Delta K_{a}, \Delta K_{c}\right)=$ $(1,1,1),(1,-1,1)$ and $(1,1,-1)$. All 3 groups of transitions are in the frequency ranges of $171-251$ and $310-506 \mathrm{GHz}$, with the coverage of $K_{a}$ and $J$ similar to those of $v_{30}=1$. The $Q$-branch 
Table 3. Changes of molecular parameters for the $v_{30}=1$ and 2 vibrational states of $g$ - $n$-PrCN obtained from our latest fit using Watson's $S$ reduction compared to the fit of Müller et al. (2016).

\begin{tabular}{|c|c|c|c|c|}
\hline $\begin{array}{l}\text { Parameters } \\
(\mathrm{MHz})\end{array}$ & $\begin{array}{c}v_{30}=1 \\
\Delta X,(\text { Müller et al. 2016) }\end{array}$ & $\begin{array}{c}v_{30}=1 \\
\Delta X, \text { this work }\end{array}$ & $\begin{array}{c}v_{30}=2 \\
\Delta \Delta X,(\text { Müller et al. 2016) }\end{array}$ & $\begin{array}{c}v_{30}=2 \\
\Delta \Delta X, \text { this work }\end{array}$ \\
\hline$A$ & $58.6419(3)$ & $58.6429(2)$ & $3.7323(5)$ & $3.7305(3)$ \\
\hline$B$ & $-7.93829(5)$ & $-7.93825(3)$ & $-0.60351(11)$ & $-0.60369(6)$ \\
\hline$C$ & $-5.17390(5)$ & $-5.17388(3)$ & $-0.15973(11)$ & $-0.15997(5)$ \\
\hline$D_{K} \times 10^{3}$ & $5.005(4)$ & $4.9987(8)$ & $0.782(5)$ & $0.775(2)$ \\
\hline$D_{J K} \times 10^{3}$ & $-0.6567(3)$ & $-0.6552(2)$ & $-0.1385(3)$ & $-0.1386(3)$ \\
\hline$D_{J} \times 10^{6}$ & $7.76(4)$ & $7.562(12)$ & $6.36(8)$ & $5.95(2)$ \\
\hline$d_{1} \times 10^{6}$ & $-8.029(10)$ & $-8.065(5)$ & $-2.455(22)$ & $-2.515(8)$ \\
\hline$d_{2} \times 10^{6}$ & $-3.605(3)$ & $-3.600(2)$ & $-0.280(7)$ & $-0.252(5)$ \\
\hline$H_{K} \times 10^{6}$ & $0.476(21)$ & $0.3743(13)$ & & $0.091(3)$ \\
\hline$H_{K J} \times 10^{9}$ & $-109.5(10)$ & $-99.2(4)$ & & $-20.52(48)$ \\
\hline$H_{J K} \times 10^{9}$ & $5.52(8)$ & $5.47(6)$ & & $1.548(49)$ \\
\hline$H_{J} \times 10^{9}$ & & $-0.0729(23)$ & & $-0.2193(17)$ \\
\hline$h_{1} \times 10^{9}$ & $-0.148(3)$ & $-0.1058(12)$ & $-0.159(5)$ & $-0.1184(10)$ \\
\hline$h_{2} \times 10^{12}$ & & $-21.98(31)$ & & $-26.26(67)$ \\
\hline$h_{3} \times 10^{12}$ & $5.0(5)$ & $13.50(13)$ & & $1.15(30)$ \\
\hline$L_{J K} \times 10^{12}$ & & $-1.015(98)$ & & \\
\hline$L_{J J K} \times 10^{15}$ & & $20.4(50)$ & & \\
\hline$L_{J} \times 10^{15}$ & & $-5.299(171)$ & & \\
\hline$l_{1} \times 10^{15}$ & & $-2.686(98)$ & & \\
\hline$\chi_{a a}$ & $-0.061(10)$ & $-0.064(10)$ & & \\
\hline$W R M S$ & 0.86 & 1.03 & & \\
\hline Highest frequency & 126597 & 500128 & 126475 & 500060 \\
\hline $\operatorname{Max} J^{\prime \prime}$ & 65 & 92 & 63 & 87 \\
\hline $\operatorname{Max} K_{a}^{\prime \prime}$ & 20 & 25 & 19 & 22 \\
\hline Transitions & 906 & 3538 & 739 & 2776 \\
\hline
\end{tabular}

Notes. The molecular parameters of $v_{30}=1$ are expressed as $X_{v=1}=X_{v=0}+\Delta X$ and for $v_{30}=2$, they are $X_{v=2}=X_{v=0}+2 \Delta X_{v_{30}=1}+\Delta \Delta X$. Numbers in parantheses represent the uncertainty compared to the last quoted digit. $\Delta \chi_{a a}$ in Müller et al. (2016) was fit on the ground-state value from Vormann \& Dreizler (1988). Other ground-state parameters used in Müller et al. (2016) were from Belloche et al. (2009). The parameters determined in this work are based on the up-dated ground-state parameters. WRMS was calculated on the fit of all lines including $v_{30}=1$ and 2 .

$b$-type transitions with $\left(\Delta J, \Delta K_{a}, \Delta K_{c}\right)=(0,1,-1)$ are all in the range $171-251 \mathrm{GHz}$ with $K_{a}^{\prime \prime}$ from $12-17, J^{\prime \prime}$ from $19-65$ and not sequential with frequency. The two sub-branches of the $Q$-branch $b$-type transitions $\left(J=K_{a}+K_{c}\right.$ and $\left.J=K_{a}+K_{c}-1\right)$ show prolate pairing and hence have doubled intensities facilitating the assignments. Taking into account that vibrational coupling may cause some transitions to shift in frequency by up to the order of $1 \mathrm{MHz}$, safe predictions can be made up to $J^{\prime \prime}=88$ at $K_{a}^{\prime \prime} \leq 19$ and $J^{\prime \prime}=59$ for $20 \leq K_{a}^{\prime \prime} \leq 25$ for $a$-type transitions, and up to $K_{a}^{\prime \prime}=14$ for $b$-type transitions.

\section{3. $v_{29}=1$ of $g-n-\operatorname{PrCN}$}

$v_{29}=1$ is a vibrational state lying in energy between $v_{30}=1$ and $v_{30}=2$. We started from the fit of Müller et al. (2016) and first added lines up to $251 \mathrm{GHz}$. When we got to $K_{a}^{\prime \prime}=8$ for new lines we started to have problems with a calculated WRMS larger than 1.40 . We noticed that $Q$-branch $b$-type lines with high $J$ numbers in Müller et al. (2016) all had residuals more than three times the attributed uncertainties $(3 \sigma)$ with the same sign for a particular $K_{a}^{\prime \prime}$. We decided to remove these problem lines from the fit, and concentrate on $Q$-branch $b$-type transitions at higher frequency which have stronger intensities (176 transitions with $K_{a}^{\prime \prime}$ between $12-17$ at $176-242 \mathrm{GHz}$ ). The newly determined parameters allowed us to make more precise predictions and identify clearly that the problem lines show internal rotation splitting, that was previously difficult to differentiate from line crowding and quadrupole hyperfine splitting. The split lines show two components separated by around $0.5 \mathrm{MHz}$ with similar intensity and center on the up-dated predictions. The center frequencies (all calculated by averaging the positions of the two components) were hence assigned with a higher uncertainty $(0.05 \mathrm{MHz})$. All lines included in our new fit up to $506 \mathrm{GHz}$ are listed in Table 14 (at the CDS). No hyperfine splitting was resolved above $171 \mathrm{GHz}$, while all lower-frequency hyperfine split lines from Müller et al. (2016) were fitted with the groundstate hyperfine structure parameters from Vormann \& Dreizler (1988). If not indicated specifically, it is the same for other states. $R$-branch $b$-type transitions were in the same three groups as $v_{30}=1$ and 2 , with the largest $K_{a}^{\prime \prime}=19$ and largest $J^{\prime \prime}=91$ near $500 \mathrm{GHz}$. The $b$-type transitions with $\left(\Delta J, \Delta K_{a}, \Delta K_{c}\right)=$ $(1,1, \pm 1)$ are prolate paired (or close) with low $J^{\prime \prime}$ and high $K_{a}^{\prime \prime}$; while $(1, \pm 1,1)$ transitions are more oblate paired with high $J^{\prime \prime}$ and low $K_{a}^{\prime \prime}$, therefore nearly half of the newly assigned, 715 $R$-branch $b$-type transitions had $\left(\Delta J, \Delta K_{a}, \Delta K_{c}\right)=(1,1,1)$. All newly assigned $R$-branch $a$-type transitions with $K_{a}^{\prime \prime}$ up to 20 and 32 were successfully fit at frequencies up to respectively 506 and $251 \mathrm{GHz}$. $a$-type transitions with $K_{a}^{\prime \prime}=11$ were badly fit above $J^{\prime \prime}=77$ for the sub-branch $J=K_{a}+K_{c}$ and $J^{\prime \prime}=82$ for $J=K_{a}+K_{c}-1$ with obvious trends. We put all these lines, with lines of larger $J^{\prime \prime}$ and $K_{a}^{\prime \prime}$ (even if some had reasonable residuals), into a separate list (Table 15 , available at the CDS) as we 
D. Liu et al.: Frequencies of the low lying vibrational states of $n$-propyl cyanide up to $506 \mathrm{GHz}$

did in $v_{30}=2$ and they were excluded from our final fit. Finally we derived the parameter list as shown in Table 4 additionally determining $\Delta H_{K}$ and $\Delta h_{1} . \Delta H_{J K}$ was decreased by $60 \%$ compared with Müller et al. (2016), because of the correction of the assignment of lines with internal rotation splitting. These corrected transition frequencies as well as the newly assigned transitions allowed us to determine $\Delta h_{1}$ and this, in turn, changed the values of $\Delta h_{2}$ and $\Delta h_{3}$ significantly. The value of $\Delta H_{J}$ was also better determined. The refined parameters allow confident predictions above $310 \mathrm{GHz}$ for $a$-type transitions with $J^{\prime \prime}$ up to 121 at $K_{a}^{\prime \prime}=10$, but less than 77 at $K_{a}^{\prime \prime}$ between 11 and 35. For $b$-type transitions, the largest $K_{a}^{\prime \prime}$ is 14 .

\section{4. $v_{28}=1$ of $g-n-\operatorname{PrCN}$}

Most transitions fitted in Müller et al. (2016) were $R$-branch $a$-type with $J^{\prime \prime}$ less than 22 and $K_{a}^{\prime \prime}$ less than 19 . The $v_{28}$ vibrational mode involves mainly methyl group torsion (Crowder 1987; Durig et al. 2001) leading to a considerable internal rotation character in the microwave spectra; some evidence was observed in Müller et al. (2016) when assigning transitions with $K_{a}^{\prime \prime} \geq 3$. Owing to this, our confident assignments for the high- $K R$-branch $a$-type transitions could not climb as high in $J^{\prime \prime}$ as the lower-energy states of $g-n$-PrCN: $J^{\prime \prime}=40$ between $K_{a}^{\prime \prime}=10-20$ at about $245 \mathrm{GHz}$. Since the internal rotation causes broadening, shoulders or splits which are more prominent in high $J$ transitions, we followed a similar procedure to Müller et al. (2016). For symmetric broadening, or splits with similar intensities of the two components with the separation around $0.6 \mathrm{MHz}$, we assigned the prediction to the center and gave an uncertainty of $0.05 \mathrm{MHz}$. For splits with an intensity ratio of $1: 2$ or $2: 1$, we assigned the prediction to the weighted average frequency and gave an uncertainty of $0.10 \mathrm{MHz}$. This is indicated in the line lists available at the CDS. The broadening and line density above $310 \mathrm{GHz}$ made secure assignments for internal-rotation affected lines impossible; finally $a$-type transitions could be fit with $K_{a}^{\prime \prime}$ up to 9 between $310-506 \mathrm{GHz}$. The small number of $b$-type transitions fitted in Müller et al. (2016) made it difficult to determine the pure $K$ parameters, $D_{K}$ and $H_{K}$. For $R$-branch $b$-type transitions, the assignments with higher $K_{a}^{\prime \prime}$ were difficult because they were close to each other. This was already the case at lower frequency as described in Müller et al. (2016). However, $309 R$-branch $b$-type transitions with $\left(\Delta J, \Delta K_{a}, \Delta K_{c}\right)=(1, \pm 1,1)$ whose $K_{a}^{\prime \prime}$ up to 9 and $J^{\prime \prime}$ up to 89 could be added to the fit up to $506 \mathrm{GHz}$. Because of the difficulty in assigning high- $K$ transitions, it was not possible to add new $(1,1,-1)$ transitions at higher frequency. Additionally, 12 new $Q$-branch $b$-type transitions could be assigned and fit; all of these lines have $K_{a}^{\prime \prime}$ between $12-17$ from $174.93-245.03 \mathrm{GHz}$. These newly assigned $b$-type transitions proved useful to determine the molecular parameters especially $\Delta D_{K}$, whose value was freed and determined consistent to the estimation in Müller et al. (2016; as Table 5). All fitted lines of $v_{28}=1$ can be found in Table 16 (available at the CDS) and additional lines were put in Table 17 (available at the CDS) as we did for other states of the gauche conformer. The limit of good predictions above $310 \mathrm{GHz}$ is $J^{\prime \prime}=89$ at $K_{a}^{\prime \prime}$ up to 5 and $J^{\prime \prime}=78$ at larger $K_{a}^{\prime \prime}(\leq 9)$ for both $a$ - and $b$-type transitions, owing to the difficulty in assigning high- $K$ transitions.

\section{5. $v_{29}=v_{30}=1$ of $g-n-\operatorname{PrCN}$}

Hirota (1962) refers to seeing combination states in his measurements but gives no further details or analysis. We used the
Table 4. Changes of molecular parameters for the $v_{29}=1$ vibrational states of $g-n$-PrCN obtained from our latest fit using Watson's $S$ reduction compared to the fit of Müller et al. (2016).

\begin{tabular}{ccc}
\hline \hline $\begin{array}{c}\text { Parameters } \\
(\mathrm{MHz})\end{array}$ & $\Delta X,($ Müller et al. 2016) & $\Delta X$, this work \\
\hline$A$ & $23.7237(5)$ & $23.7238(2)$ \\
$B$ & $2.49394(13)$ & $2.49329(5)$ \\
$C$ & $0.43186(12)$ & $0.43322(3)$ \\
$D_{K} \times 10^{3}$ & $-0.829(4)$ & $-0.8467(11)$ \\
$D_{J K} \times 10^{3}$ & $0.1557(8)$ & $0.15941(13)$ \\
$D_{J} \times 10^{6}$ & $-20.64(22)$ & $-20.188(10)$ \\
$d_{1} \times 10^{6}$ & $1.804(33)$ & $2.589(7)$ \\
$d_{2} \times 10^{6}$ & $-1.244(18)$ & $-0.984(5)$ \\
$H_{K} \times 10^{6}$ & & $-0.0773(19)$ \\
$H_{K J} \times 10^{9}$ & $42.8(41)^{a}$ & $41.48(15)$ \\
$H_{J K} \times 10^{9}$ & $-6.26(27)$ & $-2.35(2)$ \\
$H_{J} \times 10^{9}$ & $-0.524(95)$ & $-0.2729(10)$ \\
$h_{1} \times 10^{12}$ & & $-103.7(8)$ \\
$h_{2} \times 10^{12}$ & $180.0(15)$ & $-2.05(59)$ \\
$h_{3} \times 10^{12}$ & $48.1(64)$ & $1.305(198)$ \\
\hline$W R M S$ & 0.93 & 0.98 \\
Highest frequency & 126723 & 499625 \\
Max $J^{\prime \prime}$ & 52 & 90 \\
Max $K_{a}^{\prime \prime}$ & 19 & 32 \\
Transitions & 706 & $2856^{b}$ \\
\hline
\end{tabular}

Notes. The molecular parameters of $v_{29}=1$ are expressed as $X_{v=1}=$ $X_{v=0}+\Delta X$. Numbers in parantheses represent the uncertainty compared to the last quoted digit. The hyperfine structure parameters used were from Vormann \& Dreizler (1988). Other ground-state parameters used in Müller et al. (2016) were from Belloche et al. (2009). The parameters determined in this work are based on the up-dated ground-state parameters. ${ }^{(a)} \Delta H_{K J}$ had an error in sign in Müller et al. (2016) that is corrected here. ${ }^{(b)}$ We checked $288 Q$-branch $b$-type transitions used in Müller et al. (2016) and re-assigned 41 transitions with internal rotation splitting.

empirical method of estimating the parameters by addition of the changes of each individual vibrational state: $X_{v_{29}=v_{30}=1}=$ $X_{v=0}+\Delta X_{v_{30}=1}+\Delta X_{v_{29}=1}+\Delta \Delta X$. First predictions were made with $\Delta \Delta X=0$. Lines could then be easily assigned to transitions with low quantum numbers, and subsequently the correction $\Delta \Delta X$ was liberated for certain parameters to fit successively more transitions. The exploratory assignments began with intense $R$-branch $a$-type transitions and then $b$-type transitions. After all $R$-branch transitions were assigned and fitted, $Q$-branch $b$-type transitions were measured with particular care because of the already identified internal rotation of $v_{29}=1$ involved in the movement of this combination vibrational state and the higher energy giving weaker lines. For $R$-branch $a$-type transitions, we could successfully fit all transtions with $K_{a}^{\prime \prime}$ up to 20 (excluding lines of $K_{a}^{\prime \prime}=18$, which showed a clear trend in residuals between 197.48 and $221.45 \mathrm{GHz}$ ) for frequencies below $251 \mathrm{GHz}$. But in the range $310-506 \mathrm{GHz}$, the assignments appeared difficult in spite of larger intensities. As for other vibrational states of $g-n$-PrCN, the oblate pairing consisting of $a$-type and $b$-type transitions facilitated the assignments. However, the increasing separation of paired transitions with increase in quantum numbers made assignments more difficult combined with the blending and broadening in the high frequency range and the possible splits caused by internal rotations. Therefore very secure assignments with $K_{a}^{\prime \prime}$ up to 4 or 5 (for each sub-branch) 
Table 5. Changes of molecular parameters for the $v_{28}=1$ vibrational states of $g-n$-PrCN obtained from our latest fit using Watson's $S$ reduction compared to the fit of Müller et al. (2016).

\begin{tabular}{ccc}
\hline \hline Parameters & $v_{28}=1$ & $v_{28}=1$ \\
$(\mathrm{MHz})$ & $\Delta X,($ Müller et al. 2016) & $\Delta X$, this work \\
\hline$A$ & $-36.9279(135)$ & $-36.9252(53)$ \\
$B$ & $2.25206(85)$ & $2.25460(27)$ \\
$C$ & $-0.17562(44)$ & $-0.17628(15)$ \\
$D_{K} \times 10^{3}$ & $-2.2^{a}$ & $-1.96(3)$ \\
$D_{J K} \times 10^{3}$ & $0.3359(23)$ & $0.3344(6)$ \\
$D_{J} \times 10^{6}$ & $8.94(57)$ & $9.37(4)$ \\
$d_{1} \times 10^{6}$ & $-5.61(49)$ & $-6.17(3)$ \\
$d_{2} \times 10^{6}$ & $-1.19(27)$ & $-0.78(19)$ \\
$H_{K} \times 10^{6}$ & & $0.758(46)$ \\
$h_{1} \times 10^{12}$ & & $23.15(161)$ \\
$h_{2} \times 10^{12}$ & & $30.38(226)$ \\
$h_{3} \times 10^{12}$ & & $13.75(83)$ \\
\hline$W R M S$ & 0.73 & 1.05 \\
Highest frequency & 126624 & 499388 \\
Max $J^{\prime \prime}$ & 21 & 89 \\
Max $K_{a}^{\prime \prime}$ & 18 & 20 \\
Transitions & 283 & 1186 \\
\hline
\end{tabular}

Notes. The molecular parameters of $v_{28}=1$ are expressed as $X_{v=1}=$ $X_{v=0}+\Delta X$. Numbers in parantheses represent the uncertainty compared to the last quoted digit. The hyperfine structure parameters used were from Vormann \& Dreizler (1988). Other ground-state parameters used in Müller et al. (2016) were from Belloche et al. (2009). The parameters determined in this work are based on the up-dated ground-state parameters. ${ }^{(a)}$ The value of $\Delta D_{K}$ in Müller et al. (2016) was estimated with fairly secure assignments of the measured $b$-type transitions.

were first made. Even after the improvement of the parameters several lines at each $K_{a}^{\prime \prime}$ could not be correctly fitted showing systematic residuals. All these lines are in the ranges of $J^{\prime \prime}$ from 68 to 73 and from 89 to 93 . We attributed this as due coupling with other vibrational states. Finally $935 R$-branch $a$-type transitions were identified in all four frequency ranges. The assignment of $b$-type transitions was not easy due to low intensities at low frequencies and blending and broadening at high frequencies. The confidently assigned $b$-type transitions at high frequency were all oblate paired with intense $a$-type transitions with $K_{a}^{\prime \prime}$ less than 6 . Totally, $487 b$-type transitions were fitted. $Q$-branch $b$-type transitions were useful to correctly determine the parameter $D_{K}$. We identified internal rotation causing splits (with the components separated by around $0.6 \mathrm{MHz}$ ) at frequencies between $89.25-126.75 \mathrm{GHz}$, in transitions with large $J^{\prime \prime}$. In this range, $Q$-branch $b$-type transitions have $K_{a}^{\prime \prime}$ less than 11 and those that could be securely assigned have $J^{\prime \prime}$ less than 45 . For $Q$-branch $b$-type transitions with $K_{a}^{\prime \prime}$ between 13 and 18 in the range $171-251 \mathrm{GHz}$, only prolate pairs were assigned. These transitions have low $J^{\prime \prime}$ and hence are not affected by internal rotation. Finally $466 Q$-branch $b$-type transitions were correctly fitted. Moreover, 110 hyperfine split transitions were fitted with fixed hyperfine parameters from $v_{30}=1$. These are $R$-branch $a$-type transitions with $J^{\prime \prime}$ approaching $K_{a}^{\prime \prime}$ (less than 11), $b$-type transitions (both $R$-branch and $Q$-branch) with $K_{a}^{\prime \prime}$ less than 4 at frequencies below $70 \mathrm{GHz}$. The derived parameters are shown in Table 6. The fitted transitions are in Table 18 (available at the CDS), and those confidently assigned but not correctly fitted with systematic residuals are separated in Table 19 (available at the CDS). Using the newly derived molecular parameters,
Table 6. Changes of molecular parameters for the $v_{29}=v_{30}=1$ vibrational states of $g-n$-PrCN obtained from our latest fit using Watson's $S$ reduction.

\begin{tabular}{cccc}
\hline \hline Parameters & $v_{30}=1$ & $v_{29}=1$ & $v_{29}=v_{30}=1$ \\
$(\mathrm{MHz})$ & $\Delta X$ & $\Delta X$ & $\Delta \Delta X$ \\
\hline$A$ & 58.6429 & 23.7238 & $-1.50985(23)$ \\
$B$ & -7.93825 & 2.49329 & $-0.299830(42)$ \\
$C$ & -5.17388 & 0.43322 & $-0.000337(32)$ \\
$D_{K} \times 10^{3}$ & 4.9987 & -0.8467 & $0.0157(7)$ \\
$D_{J K} \times 10^{3}$ & -0.6552 & 0.15941 & $-0.01558(21)$ \\
$D_{J} \times 10^{6}$ & 7.562 & -20.188 & $-2.532(12)$ \\
$d_{1} \times 10^{6}$ & -8.065 & 2.589 & $1.601(6)$ \\
$d_{2} \times 10^{6}$ & -3.600 & -0.984 & $0.7423(22)$ \\
$H_{K} \times 10^{6}$ & 0.3743 & -0.0773 & \\
$H_{K J} \times 10^{9}$ & -99.2 & 41.48 & $2.75(60)$ \\
$H_{J K} \times 10^{9}$ & 5.47 & -2.35 & \\
$H_{J} \times 10^{9}$ & -0.0729 & -0.2729 & $0.00914(98)$ \\
$h_{1} \times 10^{12}$ & -105.8 & -103.7 & \\
$h_{2} \times 10^{12}$ & -21.98 & -2.05 & \\
$h_{3} \times 10^{12}$ & 13.498 & 1.305 & \\
$L_{J K} \times 10^{12}$ & -1.015 & & \\
$L_{J J} \times 10^{15}$ & 20.4 & & \\
$L_{J} \times 10^{15}$ & -5.299 & & \\
$l_{1} \times 10^{15}$ & -2.686 & & \\
$\chi_{a a}$ & -0.064 & & \\
WRMS & & & \\
Highest frequency & & & \\
Max $J^{\prime \prime}$ & & & \\
Max $K_{a}^{\prime \prime}$ & & & \\
Transitions & & & \\
\hline & & & \\
\hline
\end{tabular}

Notes. The molecular parameters are expressed as $X_{v_{29}=v_{30}=1}=X_{v=0}+$ $\Delta X_{v_{30}=1}+\Delta X_{v_{29}=1}+\Delta \Delta X$. Numbers in parantheses represent the uncertainty compared to the last quoted digit. Parameters without uncertainties were kept fixed in the fit. The parameters determined in this work are based on the up-dated ground-state parameters and the changes for $v_{30}=1$ and $v_{29}=1$ already determined in this work.

confident predictions of $a$-type transitions can be made up to $J^{\prime \prime}=68$ for $K_{a}^{\prime \prime} \leq 5$ below $380 \mathrm{GHz}$, and $J^{\prime \prime}=41$ for $K_{a}^{\prime \prime} \leq 41$ below $251 \mathrm{GHz}$ (excluding lines of $K_{a}^{\prime \prime}=18$ that show residuals that cannot be fitted). For $b$-type transitions, all $R$-branch predictions with $K_{a}^{\prime \prime} \leq 11$ below $251 \mathrm{GHz}$ are reliable, and $J^{\prime \prime} \leq 41$ for $K_{a}^{\prime \prime} \leq 17$ for the $Q$-branch also below $251 \mathrm{GHz}$. For frequencies above $310 \mathrm{GHz}$, the limit of $b$-type transitions has the same limit as the $a$-type because of oblate pairing.

\section{6. $v_{30}=1$ of $a-n-\operatorname{PrCN}$}

$v_{30}=1$ of $a-n-\operatorname{PrCN}$ was fit together with $v_{30}=2$ and $v_{18}=1$, in order to take into account Coriolis-type and Fermi coupling effects between the latter two states. All lines of $v_{30}=1,2$ and $v_{18}=1$ included in the final fit are given in Table 20 (available at the CDS). All confident assignments showing large residuals and a trend that could not be corrected with additional parameters were put in a separate list (Table 21, available at the CDS) as was done for the gauche conformer. Finally we obtained changes of the molecular parameters for all three vibrational states shown in Table 7 . We discuss $v_{30}=1$ first. Most transitions fitted were a-type (516 out of 540 in 
Müller et al. (2016); 1134 new transitions out of 1259 in this work) because of the low value of $\mu_{b}$. New assignments between $171-251 \mathrm{GHz}$ could be smoothly fit for all $a$-type transitions with $K_{a}^{\prime \prime}$ up to 19 , by adding $\Delta H_{J K}$. For $K_{a}^{\prime \prime}=20$, transitions with $J^{\prime \prime}$ between 38-48 between $173-217 \mathrm{GHz}$ could not be correctly assigned because of the poor line shape; assignments could be made with larger $J^{\prime \prime}$, but the residuals were already over our acceptable value $(3 \sigma)$. For assignments between $310-506 \mathrm{GHz}$, we were only able to fit up to $K_{a}^{\prime \prime}=3$ with the previous parameter list. First we tried to add $\Delta h_{1}$ and $\Delta h_{2}$, as $h_{1}$ and $h_{2}$ could be determined for the ground vibrational state but these parameters did not allow us to fit higher $K_{a}^{\prime \prime}$ lines correctly. In order to do this we had to add $\Delta h_{3}$, but in this case $\Delta h_{1}$ could not be determined any more. Finally the best fit was obtained using $\Delta h_{2}$ and $\Delta h_{3}$. For lines above $310 \mathrm{GHz}$, we could not successfully fit above $J^{\prime \prime}$ larger than 84 for $K_{a}^{\prime \prime}=14$ with all problem lines showing negatively trended residuals. Similarly $K_{a}^{\prime \prime}=15$ showed positive trended residuals and 16 positive trended but smaller residuals. The addition of high order parameters did not allow an adequate fit of these lines, and the value of parameters varied largely and became dependent on the data set used. We hence omitted them from the final fit. Although $K_{a}^{\prime \prime}=17,18$ and 19 could then be fitted with all determined parameters, by precaution we put all lines with $J^{\prime \prime}>84$ and $K_{a}^{\prime \prime}>13$ into our separate list in Table 21. Extensive fitting of $b$-type lines proved difficult. We did, however succeed in measuring $125 b$-type transitions, up to $251 \mathrm{GHz}$. Most of these $b$-type transitions are $Q$-branch with $K_{a}^{\prime \prime}$ up to 3 because of their higher intensities. For $R$-branch $b$-type transitions we could get to $J^{\prime \prime}=55$ and $K_{a}^{\prime \prime}=2$. $b$-type lines were very useful for determining $\Delta A$ and $\Delta D_{K}$, whose uncertainties were decreased to one tenth of their previous values (as Table 7). The value of $H_{K}$ in the ground-state of $a-n$-PrCN could not be correctly determined, and no differences $\left(\Delta H_{K}\right)$ were hence determined for the vibrationally excited states. Using the new parameters, predictions should be relatively confident with $J^{\prime \prime}$ up to 119 for $K_{a}^{\prime \prime} \leq 28$, but predictions for $K_{a}^{\prime \prime}=14,15$ and 16 with $J^{\prime \prime} \geq 84$ may not all be reliably precise because of the vibrational coupling. Hence the measured frequency should be used when available.

\section{7. $v_{30}=2$ and $v_{18}=1$ of $a-n-P r C N$}

The fitting for $v_{30}=2$ was started after the parameters of $v_{30}=1$ were obtained. We first fitted the transitions from $171-251 \mathrm{GHz}$ with increasing $K_{a}^{\prime \prime}$. For $K_{a}^{\prime \prime}=9$, transitions with $J^{\prime \prime}$ between 43-55 could not be correctly fit, neither could the transitions with $K_{a}^{\prime \prime}=10$ and 11. Our new assignments with higher values of $K_{a}^{\prime \prime}$ in the final line list enabled us to determine $\Delta \Delta H_{K J}$ which took a larger than estimated value of about three times $\Delta H_{K J}$. This then allowed lines with $K_{a}^{\prime \prime}$ up to 11 to be successfully fit. We then started to assign lines of $v_{18}=1$ at 171-251 GHz, since no major coupling had been observed previously up to this $K_{a}^{\prime \prime}$ value. Lines with $K_{a}^{\prime \prime}$ up to 12 could be fit for $v_{18}=1$. However, lines with higher $K_{a}^{\prime \prime}$ were calculated with residuals beyond our expectance. After including the new data, previously assigned $v_{30}=2$ lines with $K_{a}^{\prime \prime}=12$ and 13 had positive residuals above $3 \sigma$ whereas residuals with negative values could be found for previous assignments of $v_{18}=1$ with $K_{a}^{\prime \prime}=13$ and 14 . For $K_{a}^{\prime \prime}=14$ of $v_{30}=2$, residuals were between -0.22 to $-0.30 \mathrm{MHz}$ for previous assignments. These deviations are likely caused by deficiencies in describing the Coriolis-type interaction between $v_{18}=1$ and $v_{30}=2$. In contrast, the residuals for $K_{a}^{\prime \prime}=15$ of $v_{18}=1$ were positive with almost same absolute values as $v_{30}=2$. Restraining $\Delta \Delta H_{K J}$ as
Müller et al. (2016), did not help. We attributed the problem to insufficient coupling parameters, and determined that $G_{c J}$ and $F_{K}$ were necessary for fitting these lines. The effect of adding these two parameters is shown in Fig. 3. We could then fit lines of $v_{30}=2$ with $K_{a}^{\prime \prime}$ up to 19 . Lines with $K_{a}^{\prime \prime}=19$ above $J^{\prime \prime}=51$ showed asymmetrical shapes and even splits. For $v_{18}=1 \mathrm{we}$ could safely assign and fit lines with $K_{a}^{\prime \prime}$ up to 20 . We then moved on to the higher frequency range above $310 \mathrm{GHz}$. Fitting was successful for $K_{a}^{\prime \prime}=8$ for $v_{30}=2$ up to $506 \mathrm{GHz}$, but at $K_{a}^{\prime \prime}=9$ we were hindered by separation of transitions from their prolate pairs at $J^{\prime \prime}=95$, which lead to a broadening of the line shape or even unresolved splits. Fitting with higher $K_{a}^{\prime \prime}$ proved difficult, and at higher energy, coupling with other vibrational states is possible. We noticed obvious trends in the residuals of lines with $K_{a}^{\prime \prime} \geq 10$. For example, positivetrend residuals $(0.12$ to $0.31 \mathrm{MHz})$ for $J^{\prime \prime}$ between $83-111$ at $K_{a}^{\prime \prime}=10$, negative-trend residuals $(-0.08$ to $-1.46 \mathrm{MHz})$ for $J^{\prime \prime}$ between $84-109$ at $K_{a}^{\prime \prime}=11$. Lines showing identified trends in the residuals with $K_{a}^{\prime \prime}$ up to 17 are listed in Table 21; higher $K_{a}^{\prime \prime}$ lines could not be followed and assigned confidently. For $v_{18}=1$, the separation of sub-branches of $R$-branch $a$-type transitions also stopped us fitting transitions with $J^{\prime \prime}$ larger than 85 at $K_{a}^{\prime \prime}=9$. Up to $K_{a}^{\prime \prime}=16$, assignments could be correctly fit up to $J^{\prime \prime}=76$ at around $336 \mathrm{GHz}$; the fits for higher $J^{\prime \prime}$ transitions with trended residuals are included in Table 21 . We could successfully fit another $25 b$-type transitions with $K_{a}^{\prime \prime}=0$ and 1 for $v_{30}=2$ that are included in Table 20 (available at the CDS), most of them $R$-branch transitions between $171-251 \mathrm{GHz}$. For these two states, confident predictions should be possible up to $J^{\prime \prime}=110$ for $K_{a}^{\prime \prime} \leq 9$; for higher $K_{a}^{\prime \prime} \leq 17$ confident predictions are limited to $J^{\prime \prime} \leq 82$. Note that interstate transitions between $v_{30}=2$ and $v_{18}=1$ can be predicted but are too weak to be measured in laboratory and most certainly in space.

The difficulty of the fits for $v_{30}=1,2$ and $v_{18}=1$ of $a-n-\mathrm{PrCN}$ is increased by a non-resonant Coriolis interaction between $v_{30}=1$ and $v_{18}=1$. This interaction is apparent in the relatively large vibrational corrections of many spectroscopic parameters of $v_{18}=1$ and $v_{30}=2$ which are often of similar magnitude, but of opposite sign (see Table 7). Fitting of the entire present data set may thus require more changes in spectroscopic parameters than can safely be determined. Because of the multiple interactions possible between several vibrational states the determination of these parameters most likely requires substantial further measurements and analysis that are outside the scope of this work for predicting lines strong enough to be clearly seen in an astrophysical survey. However, this may be done in future.

\section{8. $v_{29}=1$ of $a-n-\operatorname{PrCN}$}

$v_{29}=1$ of the anti conformer is a vibrational state with energy between $v_{30}=2$ and $v_{18}=v_{30}=1$. The assignments for transitions in higher states were difficult since their intensities get weaker and because the possibility of coupling with other states increases. As explained previously the identification of $b$-type transitions was even harder. Since only $a$-type transitions with $\Delta K_{a}=0$ were identified it was not possible to determine $\Delta D_{K} . \Delta A$ was not so well determined. We could identify internal rotation splitting caused by the methyl group torsion with $K_{a}^{\prime \prime}=1$ and 2 (only for the sub-branch, $J=K_{a}+K_{c}$ ). All of the split lines showing similar-intensity components separated by around $0.5 \mathrm{MHz}$, are between $171-251 \mathrm{GHz}$, with $J^{\prime \prime}$ between 39-57 (for $K_{a}^{\prime \prime}=1$ ) and between 46-55 (for $K_{a}^{\prime \prime}=2$ ). 
Table 7. Changes of molecular parameters for the $v_{30}=1,2$ and $v_{18}=1$ vibrational states of $a$ - $n$-PrCN obtained from our latest fit using Watson's $S$ reduction compared to the fit of Müller et al. (2016).

\begin{tabular}{|c|c|c|c|c|c|c|}
\hline $\begin{array}{l}\text { Parameters } \\
(\mathrm{MHz})\end{array}$ & $\begin{array}{c}v_{30}=1 \\
\Delta X, \text { previously }{ }^{a}\end{array}$ & $\begin{array}{c}v_{30}=1 \\
\Delta X, \text { this work }\end{array}$ & $\begin{array}{c}v_{30}=2 \\
\Delta \Delta X, \text { previously }{ }^{a}\end{array}$ & $\begin{array}{c}v_{30}=2 \\
\Delta \Delta X, \text { this work }\end{array}$ & $\begin{array}{c}v_{18}=1 \\
\Delta X, \text { previously }{ }^{a}\end{array}$ & $\begin{array}{c}v_{18}=1 \\
\Delta X, \text { this work }\end{array}$ \\
\hline$A$ & $-810.855(12)$ & $-810.902(2)$ & $93.495(77)$ & $91.367(64)$ & $738.035(75)$ & $740.532(66)$ \\
\hline$B$ & $1.45197(7)$ & $1.45206(2)$ & $0.16373(12)$ & $0.16412(8)$ & $5.15811(20)$ & $5.15775(9)$ \\
\hline$C$ & $5.00571(6)$ & $5.00591(2)$ & $0.15867(29)$ & $0.15676(21)$ & $1.97584(33)$ & $1.97789(24)$ \\
\hline$D_{K} \times 10^{3}$ & $-263.6(80)$ & $-347.18(13)$ & & $146.83(164)$ & & $334.05(244)$ \\
\hline$D_{J K} \times 10^{3}$ & $0.4968(5)$ & $0.4936(3)$ & $0.1380(12)$ & $0.1430(8)$ & $-0.3546(11)$ & $-0.3497(11)$ \\
\hline$D_{J} \times 10^{6}$ & $7.944(31)$ & $8.115(4)$ & & $-0.381(10)$ & $-1.799(79)$ & $-2.251(8)$ \\
\hline$d_{1} \times 10^{6}$ & $1.4546(294)$ & $1.5523(7)$ & & $-0.1897(23)$ & $-1.144(87)$ & $-1.084(7)$ \\
\hline$d_{2} \times 10^{6}$ & $2.4163(244)$ & $2.6451(9)$ & & $-0.5441(32)$ & $-1.760(52)$ & $-2.152(7)$ \\
\hline$H_{K J} \times 10^{9}$ & $63.5(14)$ & $50.1(7)$ & $17^{b}$ & $158.1(43)$ & & $-59.5(79)$ \\
\hline$H_{J K} \times 10^{9}$ & & $-1.030(14)$ & & $0.171(60)$ & & $1.261(49)$ \\
\hline$H_{J} \times 10^{9}$ & & $0.0169(2)$ & & $-0.0073(5)$ & & $-0.0149(3)$ \\
\hline$h_{1} \times 10^{12}$ & & & & & & $-6.5(4)$ \\
\hline$h_{2} \times 10^{12}$ & & $-8.916(83)$ & & & & $7.45(34)$ \\
\hline$h_{3} \times 10^{12}$ & & $-6.812(48)$ & & & & $5.746(196)$ \\
\hline$L_{K K J} \times 10^{12}$ & & & & & & $-232.1(227)$ \\
\hline
\end{tabular}

\begin{tabular}{|c|c|c|c|c|c|c|}
\hline & & & & ig betwee & 1 and $v_{30}$ & \\
\hline & & & & & & \\
\hline & $-18) \times 1$ & & & & 100 & \\
\hline & $\left.30^{2}\right) \times 10$ & & & & & \\
\hline & $K\left(18,30^{2}\right)$ & & & & & \\
\hline & $\left(18,30^{2}\right)$ & & & & & \\
\hline & $\left.8,30^{2}\right) \times$ & & & & & \\
\hline & $\overline{W R M S}$ & & & & & \\
\hline Highest frequency & 126379 & 499055 & 126637 & 500496 & 126283 & 500106 \\
\hline${ }^{c} \operatorname{Max} J^{\prime \prime}$ & 28 & 114 & 28 & 113 & 28 & 112 \\
\hline${ }^{c} \operatorname{Max} K_{a}^{\prime \prime}$ & 24 & 19 & 20 & 19 & 20 & 20 \\
\hline Transition & 540 & 1799 & 575 & 1436 & 514 & 1246 \\
\hline
\end{tabular}

Notes. The molecular parameters of $v_{30}=1$ and $v_{18}=1$ are expressed as $X_{v=1}=X_{v=0}+\Delta X$ and for $v_{30}=2$, they are $X_{v=2}=X_{v=0}+2 \Delta X_{v_{30}=1}+\Delta \Delta X$. Numbers in parantheses represent the uncertainty compared to the last quoted digit. The hyperfine structure parameters used were from Vormann \& Dreizler (1988). Other ground-state parameters used in Müller et al. (2016) were from Belloche et al. (2009). The parameters determined in this work are based on the up-dated ground-state parameters. The value of $H_{K}$ in the ground-state $a-n$-PrCN cannot be correctly determined, and no differences were hence determined for the vibrationally excited states. Coupling parameters are used to fit transitions showing Coriolis and Fermi coupling of $v_{30}=2$ and $v_{18}=1$. WRMS was calculated on the fit of all lines including $v_{30}=1,2$ and $v_{18}=1$. ${ }^{(a)}$ The changes of parameters previously are from Müller et al. (2016). ${ }^{(b)}$ The value of $\Delta \Delta H_{K J}$ was estimated in Müller et al. (2016), however, we were able to free it in this fit. ${ }^{(c)}$ Only $a$-type transitions were taken into account for Max $J^{\prime \prime}$ and Max $K_{a}^{\prime \prime}$, because most transitions assigned are $a$-type for $a$ - $n$-PrCN.

(a)

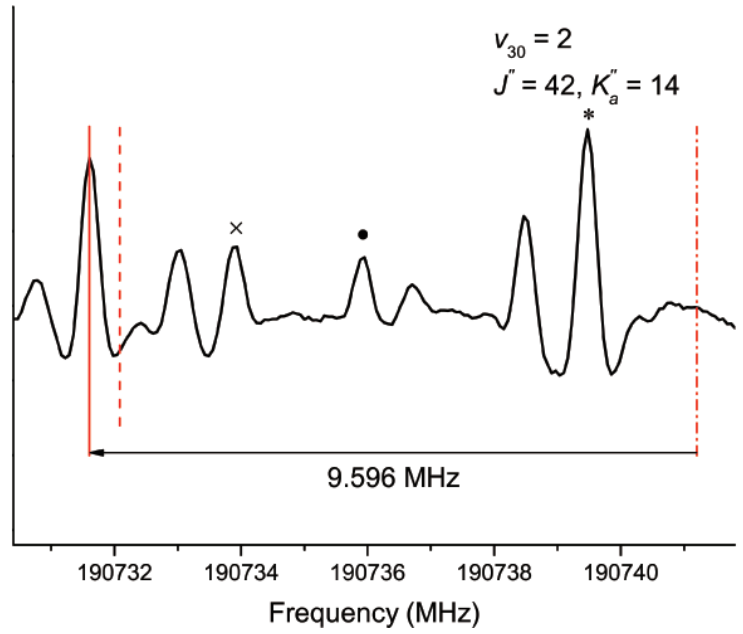

(b)

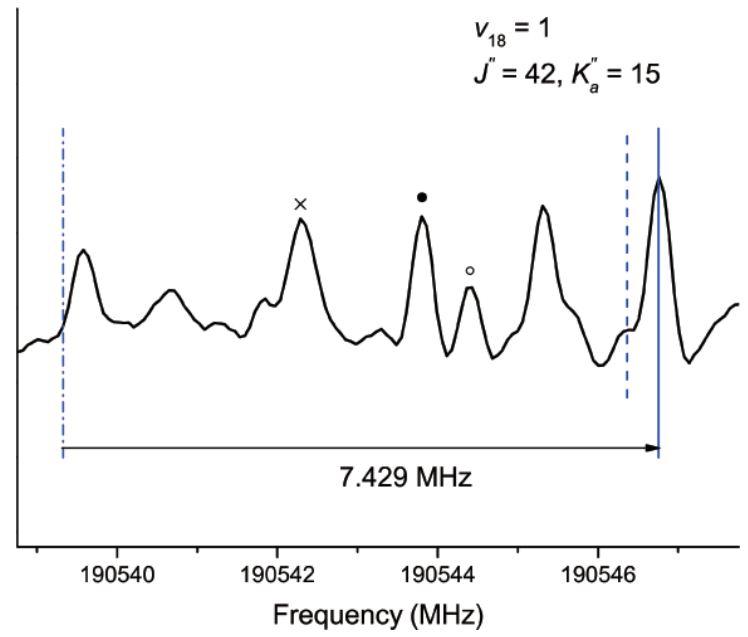

Fig. 3. Spectral extracts showing the effect of coupling parameters. The dot dash lines are predictions without coupling parameters; the dashed lines show predictions with coupling parameters from Müller et al. (2016) and the solid lines show predictions with additional coupling parameters determined in this work. Lines for other identified transitions are shown in the figures as well: $\bullet$ for ground state of $a-n$ - $\operatorname{PrCN}, *$ for $v_{30}=1$ of $a-n-\operatorname{PrCN}$, o for $v_{30}=1$ of $g-n-\operatorname{PrCN}$, and $\times$ for $v_{29}=1$ of $g-n-\operatorname{PrCN}$. 
D. Liu et al.: Frequencies of the low lying vibrational states of $n$-propyl cyanide up to $506 \mathrm{GHz}$

Table 8. Changes of molecular parameters for the $v_{29}=1$ vibrational states of $a-n-\operatorname{PrCN}$ obtained from our latest fit using Watson's $S$ reduction compared to the fit of Müller et al. (2016).

\begin{tabular}{ccc}
\hline \hline Parameters & $v_{29}=1$ & $v_{29}=1$ \\
$(\mathrm{MHz})$ & $\Delta X$, (Müller et al. 2016) & $\Delta X$, this work \\
\hline$A$ & $-0.702(137)$ & $-0.511(70)$ \\
$B$ & $-2.31897(14)$ & $-2.31911(11)$ \\
$C$ & $-1.49534(14)$ & $-1.49435(9)$ \\
$D_{J K} \times 10^{3}$ & $0.0924(9)$ & $0.1001(7)$ \\
$D_{J} \times 10^{6}$ & $-0.45(6)$ & $-0.249(9)$ \\
$d_{1} \times 10^{6}$ & & $0.266(9)$ \\
$d_{2} \times 10^{6}$ & & $0.0271(33)$ \\
$H_{K J} \times 10^{9}$ & $63.1(39)$ & $69.0(26)$ \\
$H_{J K} \times 10^{9}$ & & $3.788(91)$ \\
$H_{J} \times 10^{9}$ & & $0.01684(54)$ \\
$h_{1} \times 10^{12}$ & & $-2.72(54)$ \\
$h_{3} \times 10^{12}$ & & $2.30(20)$ \\
\hline$W R M S$ & 0.79 & 1.01 \\
Highest frequency & 126573 & 494532 \\
Max $J^{\prime \prime}$ & 28 & 114 \\
Max $K_{a}^{\prime \prime}$ & 18 & $18^{a}$ \\
Transitions & 405 & 947 \\
\hline
\end{tabular}

Notes. The molecular parameters of $v_{29}=1$ are expressed as $X_{v=1}=$ $X_{v=0}+\Delta X$. Numbers in parantheses represent the uncertainty compared to the last quoted digit. The hyperfine structure parameters used were from Vormann \& Dreizler (1988). Other ground-state parameters used in Müller et al. (2016) were from Belloche et al. (2009). The parameters determined in this work are based on the up-dated ground-state parameters. ${ }^{(a)}$ Max $K_{a}^{\prime \prime}$ of newly assigned transitions is 17 ; transitions with $K_{a}^{\prime \prime}=18$ are all fitted by Müller et al. (2016) below $125 \mathrm{GHz}$.

At higher frequencies internal rotation splitting and accidental line overlap could not be discriminated and hence assignments were not made if a single symmetric line was not identified. Finally, transitions with $K_{a}^{\prime \prime}$ up to 17 could be fit between $171-251 \mathrm{GHz}$ with $J^{\prime \prime}$ between 39 and 58 . Above $171 \mathrm{GHz}$ transitions with $K_{a}^{\prime \prime}=11$ could not be correctly fit, with residuals from 0.43 to $1.53 \mathrm{MHz}$ showing a trend with increasing $J^{\prime \prime}$. This is probably due to coupling with another vibrational state, as other $K_{a}^{\prime \prime}$ transitions (above and below) in the frequency range could be well fitted. For assignments from $310-506 \mathrm{GHz}$, we could fit transitions with $K_{a}^{\prime \prime}$ up to 8 . For weaker none-prolate paired transitions with $K_{a}^{\prime \prime}$ less than 8 , overlaps stopped us fitting more transitions, especially those with $K_{a}^{\prime \prime}$ from 4 to 7. Higher $K_{a}^{\prime \prime}$ transitions were assigned but all with trends in the residuals, and were removed to the supplementary list given in Table 23 (available at the CDS) with all assigned $K_{a}^{\prime \prime}=11$ transitions not included in the fit. Explorative fits including transitions with $K_{a}^{\prime \prime}=9$ and 10 at $310-506 \mathrm{GHz}$ changed determined parameters significantly and required illogical higher order parameters. Also the fits became dataset dependent since the newly determined parameters fit transitions with even higher $K_{a}^{\prime \prime}$, worse than the previous. Therefore all assignments of transitions with $K_{a}^{\prime \prime} \geq 9$ were moved to Table 23 leaving transitions included in the fit in Table 22 (available at the CDS). Finally the changes of parameters are shown in Table 8 . Confident predictions can be made with $K_{a}^{\prime \prime} \leq 8$ with good results (see section 3.1) for $J^{\prime \prime}$ up to 90. Higher $K_{a}^{\prime \prime}$ predictions can be made with relative confidence especially at lower frequencies except for $K_{a}^{\prime \prime}=11$ because of the unidentified vibrational coupling.
Table 9. Changes of molecular parameters for the $v_{18}=v_{30}=1$ vibrational states of $a-n$-PrCN obtained from our latest fit using Watson's $S$ reduction.

\begin{tabular}{cccc}
\hline \hline Parameters & $v_{30}=1$ & $v_{18}=1$ & $v_{18}=v_{30}=1$ \\
$(\mathrm{MHz})$ & $\Delta X$ & $\Delta X$ & $\Delta \Delta X$ \\
\hline$A$ & -810.902 & 740.532 & $-196.258(60)$ \\
$B$ & 1.45206 & 5.15775 & $-0.42142(7)$ \\
$C$ & 5.00591 & 1.97789 & $0.09133(9)$ \\
$D_{K} \times 10^{3}$ & -347.2 & 334.05 & $-304.5(44)$ \\
$D_{J K} \times 10^{3}$ & 0.4936 & -0.3497 & $-0.3470(9)$ \\
$D_{J} \times 10^{6}$ & 8.115 & -2.251 & $0.4526(134)$ \\
$d_{1} \times 10^{6}$ & 1.5523 & -1.084 & $0.3759(33)$ \\
$d_{2} \times 10^{6}$ & 2.6451 & -2.152 & $1.5351(62)$ \\
$H_{K J} \times 10^{9}$ & 50.1 & -59.5 & \\
$H_{J K} \times 10^{9}$ & -1.03 & 1.26 & \\
$H_{J} \times 10^{9}$ & 0.0169 & -0.0149 & $-0.00851(65)$ \\
$h_{1} \times 10^{12}$ & & -6.5 & \\
$h_{2} \times 10^{12}$ & -8.916 & 7.45 & \\
$h_{3} \times 10^{12}$ & -6.812 & 5.746 & $-3.00(39)$ \\
$L_{K K J} \times 10^{12}$ & & -232.1 & \\
\hline$W R M S$ & & 1.12 & \\
Highest frequency & & & 492190 \\
Max $J^{\prime \prime}$ & & & 113 \\
Max $K_{a}^{\prime \prime}$ & & & 613 \\
Transitions & & &
\end{tabular}

Notes. The molecular parameters are expressed as $X_{v_{18}=v_{30}=1}=X_{v=0}+$ $\Delta X_{v_{30}=1}+\Delta X_{v_{18}=1}+\Delta \Delta X$. Numbers in parantheses represent the uncertainty compared to the last quoted digit. Parameters without uncertainties were kept fixed in the fit. The hyperfine structure parameters used were from Vormann \& Dreizler (1988). The parameters determined in this work are based on the up-dated ground-state parameters and the changes for $v_{30}=1$ and $v_{18}=1$ already determined in this work.

\section{9. $v_{18}=v_{30}=1$ of $a-n-\operatorname{PrCN}$}

We present in Table 9 the first published molecular parameters for $v_{18}=v_{30}=1$ of $a-n$-PrCN. Hirota (1962) refers to seeing combination states in his measurements but gives no further details or analysis. The assignments were quite difficult as this state has the highest energy in our present study. Moreover, just as $v_{18}=1$ and $v_{30}=2$ interact resonantly, $v_{18}=v_{30}=1$ and $v_{30}=3$ are expected to interact resonantly, and $v_{18}=v_{30}=1$ is expected to interact at least non-resonantly with $v_{30}=2$ and with $v_{18}=2$. Finally 613 transitions (492 lines) were assigned and fitted below $506 \mathrm{GHz}$ (Table 24, available at the CDS). All transitions assigned are $a$-type $R$-branch because of their strong intensities. At the beginning, only parameters of $v_{30}=1$ and $v_{18}=1$ were used to make predictions, that can be seen from the table to give relatively correct estimates of $B, C$ and $D_{J}$. These estimates were sufficiently correct, along with the other approximate parameters, to identify lines with $K_{a}^{\prime \prime}$ between 0 and 5 below $70 \mathrm{GHz}$. Then more lines could be identified with iterative fitting and refinement of the parameters. Lines were assigned sequentially through all four available frequency ranges: 36-70, 89-126, 171-251, and 310-506 GHz as increasing $J^{\prime \prime}$ and $K_{a}^{\prime \prime}$. We could safely assign transitions with the highest $K_{a}^{\prime \prime}=8$ in all available frequency ranges. Below $70 \mathrm{GHz}$ we were able to assign some additional lines ( $J^{\prime \prime}$ between 10 and 14 for $K_{a}^{\prime \prime}=9$; $J^{\prime \prime}$ between 11 and 13 for $K_{a}^{\prime \prime}=10$ ). Assignments for higher $K_{a}^{\prime \prime}$ transitions need high order centrifugal distortion parameters, which are also better determined by fitted transitions with high 
quantum numbers. Lower intensities of higher $K_{a}^{\prime \prime}$ transitions and possible coupling with $v_{29}=1$ presented ambiguities for their assignments. Conversely lack of lines with high $K_{a}^{\prime \prime}$ make it difficult to characterize any coupling with $v_{29}=1$. Exploratory fits for transitions with $K_{a}^{\prime \prime}=9$ and 10 between $89-126 \mathrm{GHz}$ resulted in negative residuals of about $-0.2 \mathrm{MHz}\left(20 \leq J^{\prime \prime} \leq 22\right)$ and $-0.36--0.45 \mathrm{MHz}\left(20 \leq J^{\prime \prime} \leq 24\right)$ respectively, however without any clear trends; all these tentative assignments are listed in Table 25 (available at the CDS). Other assignments fitted with significant residuals (showing that more parameters are needed), include lines with $51 \leq J^{\prime \prime} \leq 55$ of $K_{a}^{\prime \prime}=8$ at 230.61-248.34 GHz (residuals between $0.09-0.34 \mathrm{MHz}$ ) and $90 \leq J^{\prime \prime} \leq 111$ of $K_{a}^{\prime \prime}=1$ at $396.84-486.70 \mathrm{GHz}$ (residuals between $-0.16--0.36 \mathrm{MHz}$ ). The WRMS we derived was somewhat larger than other states because high frequency lines were somewhat less well predicted with the current parameter list. We note that including $\Delta \Delta H_{K J}$ in the fit gave a determined value of $-547(43) \times 10^{-9}$, however, since this value was unreasonably large we did not use it in the final fit. When $J$ approaches $K_{a}$, broadening and splitting caused by quadrupole hyperfine splitting were identified. 58 hyperfine split transitions below $70 \mathrm{GHz}$ were added to the list with $K_{a}^{\prime \prime}$ between four and ten. Hyperfine structure parameters from Vormann \& Dreizler (1988) for the ground state are sufficient to fit them. Confident predictions are possible up to $K_{a}^{\prime \prime}=7$, for $J^{\prime \prime} \leq 89$. For $K_{a}^{\prime \prime}=8$, up to $J^{\prime \prime}=50$ and for $K_{a}^{\prime \prime}=9$ and 10 , only predictions below $70 \mathrm{GHz}$ can be treated with full confidence.

\section{Conclusion and outlook}

We have added 11453 new lines to fits for both conformers of $n$-PrCN, mainly in the frequency ranges $171-251$ and 310-506 GHz. We have hence improved molecular parameters for the ground states of both anti- and gauche-n-propyl cyanide and for excited vibrational states of the gauche conformer $\left(v_{30}=\right.$ $\left.1, v_{29}=1, v_{30}=2, v_{28}=1\right)$ and anti conformer $\left(v_{30}=1\right.$, $\left.v_{18}=1, v_{30}=2, v_{29}=1\right)$. The inclusion of these newly assigned lines gives a more precise and extended list of molecular parameters to improve the predictions over the entire operating range of ALMA. The present data should be useful to search for vibrationally excited states of $n$-PrCN in Orion KL with ALMA. For Coriolis and Fermi coupling between $v_{30}=2$ $\left(K_{a}^{\prime \prime} \geq 13\right)$ and $v_{18}=1\left(K_{a}^{\prime \prime} \geq 14\right)$, two more coupling parameters $F_{K}$ and $G_{C J}$ were derived from fitting. During the analysis, we not only continued assigning internal rotation split transitions in $v_{28}=1$ of $g-n$-PrCN between $171-251 \mathrm{GHz}$ but also identified additional internal rotation splitting of the $Q$-branch $b$-type transitions occuring in $v_{29}=1$ (for both the fundamental and combination states) of the gauche conformer at frequencies below $125 \mathrm{GHz}$ and also in the $R$-branch $a$-type transitions in $v_{29}=1$ of the anti conformer with $K_{a}^{\prime \prime}=1$ and 2 , at $171-251 \mathrm{GHz}$. This splitting is not expected to be resolved in astrophysical spectra. We gave the first published measurements and derived parameters for the combination state $v_{18}=v_{30}=1$ for $a-n-\operatorname{PrCN}$ and for the combination state $v_{29}=v_{30}=1$ for $g-n$-PrCN, both found by using our parameters for the individual vibrational states. Transitions of these and other higher lying vibrational states may be observable in the new EMoCA data obtained in ALMA Cycle 4. After each state and conformer, the reliability of predictions is discussed in particular as regards vibrational coupling, and limits of quantum numbers and frequencies are given. No problems have been found with the ground states. For the vibrational states above the limits given, it is best to use the measured frequencies given in the CDS when available. Measurements are presently not available in the ranges: 70-89, 127-171, and 251-310 GHz. As most of the perturbations observed are above about $350 \mathrm{GHz}$, confident predictions are available in these gaps. However, there are some particular cases. For $v_{29}=1$ of the anti conformer, lines of $K_{a}^{\prime \prime}=11$ are perturbed above $176 \mathrm{GHz}$ hence no confident frequencies are available in the highest frequency gap. Similarly for the combination state $v_{29}=v_{30}=1$ of the gauche conformer, for $K_{a}^{\prime \prime}=18$ there are no confident frequencies available in the highest frequency gap. For the combination state $v_{18}=v_{30}=1$ of the anti conformer, confident data is not available if not measured above $K_{a}^{\prime \prime}=8$ for frequencies above $70 \mathrm{GHz}$, and for $K_{a}^{\prime \prime}=8$ for frequencies above $221 \mathrm{GHz}$.

Lines assigned but not fitted correctly with our up-dated parameters are given as separate lists for astrophysical identification and will require future work to identify other vibrational couplings. These lines either have a shift more than three times the estimated experimental uncertainty or show clear trends in deviation, or both. Also lines that fit correctly but above $K_{a}$ and $J$ ranges already showing deviations have been included in the supplementary list and not in the fit by precaution. There is most likely in $a-n$-PrCN, a non-resonant interaction between $v_{30}=1$ and $v_{18}=1$ as indicated by large but opposite variations in the determined $\Delta A$ rotational constant. $v_{30}=2$ is also probably affected by coupling with nearby states. Hence full treatment of the perturbed lines most likely requires the measurement at higher sensitivity of several additional higher vibrational states such as $v_{30}=3$ and $v_{18}=2$ by combined analysis. Tentative attempts to identify transitions of $v_{30}=3$ in both conformers by extrapolation of the parameters from $v_{30}=0,1$ and $v_{30}=2$ have not as yet been successful and require further work. Measurements of gas-phase rovibrational spectra in the far infrared may also provide useful data for a more extensive analysis. Evidence for coupling in the gauche conformer is also seen but affects less the fitting. In general fitting of the gauche conformer is easier because the higher asymmetry allows more parameters to be determined independently.

Acknowledgements. The work in Cologne was supported by the Deutsche Forschungsgemeinschaft (DFG) through the collaborative research grant SFB 956 project B3 and through the Gerätezentrum "Cologne Center for Terahertz Spectroscopy". D.L. and A.W. thank PCMI for funding visits to Cologne for measurements and discussion with collaborators. PCMI is the French program "Physique et Chimie du Milieu Interstellaire" funded by the Conseil National de la Recherche Scientifique (CNRS) and Centre National d'Etudes Spatiales (CNES). They also thank the DFG via SFB 956 project B3 for funding additional-visits to Cologne. D.L. thanks the Chinese Scholarship Council (CSC) for funding his $\mathrm{PhD}$ study in France.

\section{References}

Avery, L., Bell, M., Cunningham, C., et al. 1994, ApJ, 426, 737

Belloche, A., Garrod, R., Müller, H., et al. 2009, A\&A, 499, 215

Belloche, A., Müller, H. S., Menten, K. M., Schilke, P., \& Comito, C. 2013, A\&A, 559, A47

Belloche, A., Garrod, R. T., Müller, H. S., \& Menten, K. M. 2014, Science, 345, 1584

Belloche, A., Müller, H., Garrod, R., \& Menten, K. 2016, A\&A, 587, A91

Cernicharo, J., Agúndez, M., Kahane, C., et al. 2011, A\&A, 529, L3

Clark, F., Brown, R., Godfrey, P., Storey, J., \& Johnson, D. 1976, ApJ, 210, L139 Crowder, G. 1987, J. Mol. Struct., 158, 229

Daly, A. M., Bermúdez, C., López, A., et al. 2013, ApJ, 768, 81

Demaison, J., \& Dreizler, H. 1982, Z. Naturforsch. A, 37, 199

Demyk, K., Wlodarczak, G., \& Carvajal, M. 2008, A\&A, 489, 589

Durig, J., \& Li, Y. 1974, J. Mol. Struct., 21, 289

Durig, J. R., Drew, B. R., Koomer, A., \& Bell, S. 2001, Phys. Chem. Chem. Phys., 3, 766

Fortman, S. M., McMillan, J. P., Neese, C. F., et al. 2012, J. Mol. Spectrosc., 280,11 
D. Liu et al.: Frequencies of the low lying vibrational states of $n$-propyl cyanide up to $506 \mathrm{GHz}$

Friedel, D. N., Snyder, L., Remijan, A. J., \& Turner, B. 2005, Astrophys. J. Lett., 632, L95

Gibb, E., Nummelin, A., Irvine, W. M., Whittet, D., \& Bergman, P. 2000, ApJ, 545,309

Goldsmith, P., Krotkov, R., Snell, R., Brown, R., \& Godfrey, P. 1983, ApJ, 274, 184

Groesbeck, T., Phillips, T., \& Blake, G. A. 1994, Astrophys. J. Suppl. Ser., 94, 147

Guélin, M., Cernicharo, J., Navarro, S., et al. 1987, A\&A, 182, L37

Guilloteau, S., Omont, A., \& Lucas, R. 1987, A\&A, 176, L24

Herberich, G. E. 1967, Z. Naturforsch. A, 22, 543

Hirota, E. 1962, J. Chem. Phys., 37, 2918

Hollis, J., Lovas, F., Suenram, R., Jewell, P., \& Snyder, L. 1983, ApJ, 264, 543

Kobayashi, K., Ogata, K., Tsunekawa, S., \& Takano, S. 2007, Astrophys. J. Lett., 657, L17

Kolesniková, L., Alonso, E., Mata, S., Cernicharo, J., \& Alonso, J. 2017, Astrophys. J. Suppl. Ser., 233, 24

Lovas, F., Suenram, R., Snyder, L., Hollis, J., \& Lees, R. 1982, ApJ, 253, 149

Lucas, R., \& Cernicharo, J. 1989, A\&A, 218, L20

Mauersberger, R., Henkel, C., \& Wilson, T. 1988, A\&A, 205, 235

Mehringer, D. M., Pearson, J. C., Keene, J., \& Phillips, T. G. 2004, ApJ, 608 306

Motiyenko, R., Tercero, B., Cernicharo, J., \& Margulès, L. 2012, A\&A, 548 A71

Müller, H. S., Coutens, A., Walters, A., Grabow, J.-U., \& Schlemmer, S. 2011, J. Mol. Spectrosc., 267, 100

Müller, H. S., Walters, A., Wehres, N., et al. 2016, A\&A, 595, A87

Pagani, L., Favre, C., Goldsmith, P., et al. 2017, A\&A, 604, A32

Schilke, P., \& Menten, K. M. 2003, ApJ, 583, 446

Schilke, P., Güsten, R., Schulz, A., Serabyn, E., \& Walmsley, C. 1992, A\&A, 261, L5

Schilke, P., Mehringer, D. M., \& Menten, K. M. 1999, Astrophys. J. Lett., 528, L37
Solomon, P., Jefferts, K., Penzias, A., \& Wilson, R. 1971, ApJ, 168, L107 Thorwirth, S., Wyrowski, F., Schilke, P., et al. 2003, ApJ, 586, 338

Turner, B. 1987a, A\&A, 183, L23

Turner, B. 1987b, A\&A, 182, L15

Vormann, K., \& Dreizler, H. 1988, Z. Naturforsch. A, 43, 338

Wlodarczak, G., Martinache, L., Demaison, J., Marstokk, K.-M., \& Møllendal, H. 1988, J. Mol. Spectrosc., 127, 178

Yamamoto, S., Saito, S., Guélin, M., et al. 1987, ApJ, 323, L149

Ziurys, L., \& Turner, B. 1986, ApJ, 300, L19

\section{Appendix A: Line lists at the CDS}

Line lists availabe at the CDS include: fitted transitions at 36-70, $89.25-126.75,171-251$ and $310-506 \mathrm{GHz}$ for $a-n-\mathrm{PrCN}$ in the ground state (Table 10), $v_{30}=1, v_{30}=2$ and $v_{18}=1$ (Table 20), $v_{29}=1$ (Table 22) and $v_{18}=v_{30}=1$ (Table 24); for $g-n-\operatorname{PrCN}$ in the ground state (Table 11), $v_{30}=1, v_{30}=2$ (Table 12), $v_{29}=1$ (Table 14), $v_{28}=1$ (Table 16) and $v_{29}=v_{30}=1$ (Table 18). Additionally, a smaller number of lines confidently assigned but not fitted correctly and showing systematic residuals indicating as yet uncharacterized vibrational coupling are given in separate lists. The lines that could be correctly fitted but are above quantum number limits first showing systematic residuals are also placed in these separate lists and not used in the final fits by precaution. The separate lists include for $g-n-\operatorname{PrCN}$, $v_{30}=2$ (Table 13), $v_{29}=1$ (Table 15), $v_{28}=1$ (Table 17) and $v_{29}=v_{30}=1$ (Table 19); and for $a-n-\operatorname{PrCN}, v_{30}=1, v_{30}=2$ and $v_{18}=1$ (Table 21), $v_{29}=1$ (Table 23) and $v_{18}=v_{30}=1$ (Table 25). 